Sharif University of Technology
Scientia Iranica
Transactions E: Industrial Engineering
IRANtp://scientiairanica.sharif.edu

\title{
An uncertain allocation model for data envelopment analysis: A case in the Iranian stock market
}

\author{
M. Jamshidi ${ }^{a}$, M. Sanei $i^{a, *}$, and A. Mahmoodirad ${ }^{b}$ \\ a. Department of Mathematics, Central Tehran Branch, Islamic Azad University, Tehran, Iran. \\ b. Department of Mathematics, Masjed-Soleiman Branch, Islamic Azad University, Masjed-Soleiman, Iran. \\ Received 16 December 2019; received in revised form 5 September 2020; accepted 2 November 2020
}

\section{KEYWORDS \\ Data envelopment analysis; \\ Uncertainty theory; \\ Belief degree; \\ Stock market.}

\begin{abstract}
Data Envelopment Analysis (DEA) can be employed to evaluate the operation of units as one of the most important concerns of managers. DEA is a linear programming technique for calculating relative performance of Decision-Making Units (DMUs) with multiple input and output. However, although all the input and output are considered as certain items in these models, there are uncertain items in the real word and the existing interference between these two concepts will result in uncertain models. Allocation models were studied in an uncertain environment with belief degree-based uncertain input costs and output prices. Belief degree-based uncertainty is useful for cases in which there is no historical information on an uncertain event. Utilizing the uncertain entropy model as a second objective function, the cost and revenue models showed an optimal performance with the maximum dispersion rate in their constituent components. As a solution methodology, the uncertain allocation models were separately converted into crisp models by the Expected Value (EV) and the Expected Value and Chance-Constrained (EVCC) methods. A practical example from the Iranian stock market was also employed to evaluate the performance of the new model.
\end{abstract}

(C) 2022 Sharif University of Technology. All rights reserved.

\section{Introduction}

Data Envelopment Analysis (DEA) is recognized as a robust analytical tool extensively utilized in measuring the relative efficiency of a group of Decision-Making Units (DMUs) with multiple input and output. The DEA model, originally named CCR, was first developed by Charnes et al. [1] on a printed paper. They expanded the nonparametric method introduced by Farrell [2] to gauge DMUs with multiple input and output. CCR makes use of mathematical optimiza-

*. Corresponding author.

E-mail address: masoudsanei49@yahoo.com (M. Sanei)

doi: $10.24200 /$ sci.2020.55047.4046 tion in a linear programming formula to measure the performance of a DMU relative to a set of DMUs. Afterwards, Banker et al. [3] introduced the BCC model. In addition to CCR and BCC, several models have been developed that discuss DEA from several perspectives. Instances are RAM by Cooper et al. [4], slack-adjust by Sueyoshi [5], additive model by Seiford and Thrall [6], Slacks-Based Measure (SBM) model by Tone [7], and FDH model by Deprins et al. [8], all of which are DEA-based models.

In classical DEA models, DMUs are evaluated by considering input and output values in order to measure rational efficiency as compared to different DMUs. Eventually, the measure to which rational efficiency belongs is obtained between $(0,1)$. DEA is used for measuring and analyzing such concepts as 
cost and revenue efficiencies [9]. In fact, one of the most important aspects of product analysis and organization is to measure cost and revenue efficiencies [2]. Real efficiency models for calculating cost efficiency search for a unit that requires the lowest cost for buying input not more than the input to the units under investigation in order to produce output equal to the output of the units under investigation. Furthermore, a revenue efficiency model searches for a unit which gains the best revenue from selling output greater than output of the units under investigation by consuming the input equal to the input of the units under investigation.

The method for experimental evaluation of cost and revenue efficiencies was first developed by Färe et al. [10]. In the cost and revenue model introduced by Färe et al. [10], not only the modeling needs input and output, but also every price can be different in every unit, which may limit the applications of the model. Their model was based on some sampling hypotheses. The input should be homogenous and the prices should be available and specified. Because of changes in the process or in input specifications, the techniques and input in big and small organizations might be different. As a result, input and their costs could also be different. Accordingly, the technical structure of the DEA model could be more sophisticated [9].

According to Jamshidi et al. [11], the classical DEA models assumed that input and output were represented by precise values. However, in many real-world systems like banking, insurance, and other financial systems, the input and output are not precise and cannot be measured exactly. Considering this, many researchers tried to formulate DEA problems with different hypotheses. A possible hypothesis is the earliest principles, which may be used to build stochastic DEA models. Sengupta [12] summed up the stochastic DEA model utilizing the Expected Value (EV). Moreover, Banker [13] consolidated the elements of applied mathematics under DEA in order to develop a statistical method. Several papers utilized chanceconstrained programming for DEA so as to introduce stochastic varieties to information [4,14-16]. Fuzzy outlook is another theory the hypothesis of which has been utilized to cope with the uncertainty in DEA. Cooper et al. [17-19] introduced a technique to deal with inaccurate information such as moderate data, adjectival data, and ratio moderate data in DEA. Furthermore, Kao and Liu [20] designed a technique to discover the membership function of fuzzy performance marks when each input and output was a fuzzy number. Entani et al. [21] proposed an interval potency DEA model by pessimistic and idealistic values. Several researchers have introduced the possibility measure into DEA [22-24]. As confirmed by several studies, human uncertainty does not come with the same fuzziness in all cases. To overcome the shortcomings of the fuzzy theory, Liu [25] introduced the uncertain theory and refined it in 2010 as an understandable mathematical structure for confronting uncertainty in data, which served as a strong alternative to the probability theory when one had to restrict the information in the face of insufficient trusted data. The belief degree function is associated with an underlying concept of this theory built according to the opinion of the experts [26].

Optimization problems with uncertain data can be even more interesting and realistic in uncertain environments with uncertain values for parameters and variables [27]. To deal with uncertain parameters, any approach based on randomness, fuzzy theory, stochastic programming, probability theory, and so on can be applied to the historical information on the parameters.

In such cases, the uncertain manner of the problem is estimated from the historical data as a probability function, random number, fuzzy number, etc. [25]. On the other hand, for cases in which no historical information on an uncertain event exists, uncertainty theory based on belief degree has been employed to solve the problem.

The uncertainty theory can be explained by a simple example. Consider a bridge with undetermined strength. At first, it is assumed that no destructive experiment is allowed on the bridge. Thus, there is no sample regarding the strength of the bridge. In this case, no statistical methods exist for estimating its probability distribution. Therefore, there is no choice but to invite bridge engineers to evaluate the belief degrees about the strength of the bridge [28]. Some basic concepts of the belief degree-based uncertainty theory will be explained in Section 2 and a complete study of this topic can be found in Liu [25].

The belief degree depends heavily on personal knowledge (including preferences) concerning an event. When the personal knowledge changes, the belief degree changes as well. Different people may offer different belief degrees. The question is which belief degree is correct and it may be answered as follows: all belief degrees are wrong, but some are useful [28].

A belief degree becomes "correct" only when it is close enough to the frequency of the indeterminate quantity, which, however, does not usually occur. Numerous surveys have demonstrated that human beings usually estimate a much wider range of values than the object actually takes. This human conservatism makes the belief degrees deviate far from the frequency. Thus, all belief degrees are wrong compared with the real frequency [25]. Nevertheless, it is undeniable that these belief degrees are indeed helpful for decision-making. Moreover, as determined by the choice of $\alpha$, there is a risk that DMUs would not be efficient even when their condition is satisfied [28].

Wen et al. [29] applied the uncertain theory 
for the first time to rewriting the DEA model in uncertainty condition and then, published a paper on the sensitivity and stability of the additive model in terms of uncertainty. Wen et al. [30] also introduced a new additive model with uncertain input and output. Additionally, Wen et al. [31] developed the DEA model with uncertainty index ranking for criteria. Lio et al. [32] also published a paper to evaluate DMUs with uncertain input and output. Jamshidi et al. [11] developed the RUSSEL DEA model with uncertain input and output. Jamshidi et al. [26] applied the SBM model to an uncertain environment where the uncertain input and output were belief degree-based uncertainty.

These studies are nevertheless insufficient for describing programming with uncertain data [27,3339 ] and new models are often introduced to create new methods.

Based on the Shannon entropy, Liu [40] introduced the concept of entropy for the first time to determine the uncertainty of uncertain variables resulting from information deficiency. Clearly, entropy functions are used as a means for measuring dispersion in allocation models. Chen and Dai [41] and Dai and Chen [42] investigated the maximum entropy of uncertainty distribution for uncertain variables. They presented the entropy of a function of an uncertain variable. In the present study, input costs and output prices are considered as uncertain variables and then, two different models are proposed to convert the new model into a crisp model to deal with the uncertainty problem. Thereafter, uncertain allocation models are used in a practical example in the Iranian stock market to find a stock portfolio with the maximum return.

Based on the literature on the uncertain DEA problems, no serious study has been performed on incorporating entropy and belief degree-based uncertain DEA models. In fact, entropy measures the degree of uncertainty. This study for the first time aims at dealing with allocation models in an uncertain environment. Since entropy helps with the diversification of the model, another function is considered as a second objective function. This in turn results in the best and highest diversification of allocation models. Considering the maximum entropy in allocation models helps us achieve the highest revenue and the lowest cost with a maximum dispersal measure. This new multi-objective model will result in higher efficiency of units, which are considered more dispersed in all processes. The parameters of the objective function and constraints are considered to be of zigzag uncertainty variables. Two approaches of $\mathrm{EV}$ and Expected Value and Chance-Constrained (EVCC) methods are developed for the uncertain allocation problems. A real practical example from the Iranian stock market is also presented to evaluate the performance of the new model. The new multi-objective uncertain allocation models will enhance the power of managerial decisions and offer a clear way for choosing components of allocation models.

The paper is organized as follows: Some preliminary knowledge on uncertainty theory is reviewed in Section 2. Entropy function in an uncertain space is introduced in Section 3. Basic concepts of the allocation DEA models are introduced in Section 4. Some new uncertain DEA models are introduced in Section 5 and their new structures are proven. Section 6 concerns crisp equivalents of the DEA models. Entropy-based allocation models with uncertain variables are introduced in Section 7. The weighted method, which is solely a multi-objective model, is introduced in Section 8. Finally, an applied example to the Iranian stock market is solved by the allocation uncertain models.

\section{Preliminaries}

Here, the basic concepts and presented uncertain variables are discussed [25]. Let $\Gamma$ be a nonempty set and $L$ an $\sigma$-algebra over $\Gamma$. Each element $\Lambda \in L$ is called an event. A set function $\mathcal{M}\{\Lambda\} \in[0,1]$ is known as an uncertain measure if it satisfies the following three axioms [25]:

1. $\mathcal{M}\{\Lambda\}=1$ for the universal set $\Gamma$;

2. $\mathcal{M}\{\Lambda\}+\mathcal{M}\left\{\Lambda^{c}\right\}=1$ for any event $\Lambda$;

3. For every countable sub-additive of events $\left\{\Lambda_{i}\right\}$, we have $\mathcal{M}\left\{\bigcup_{i=1}^{\infty} \Lambda_{i}\right\} \leq \sum_{i=1}^{\infty} \Lambda_{i}$.

Definition 1 [25]. The set function $\mathcal{M}$ is called an uncertain measure if it meets the duality, normality, and subadditivity axioms.

The uncertain measure has the following attributes:

i. $\mathcal{M}\{\phi\}=0$;

ii. $0 \leq \mathcal{M}\{\Lambda\} \leq 1$ for any event $\Lambda$;

iii. $\mathcal{M}\left\{\Lambda_{1}\right\} \leq \mathcal{M}\left\{\Lambda_{2}\right\}$ for any events $\Lambda_{1} \subset \Lambda_{2}$.

The triplet $(\Gamma, L, \mathcal{M})$ is called an uncertainty space. In order to define the product uncertain measure, Liu [43] proposed the fourth axiom as follows: Let $\left(\Gamma_{k}, L_{k}, \mathcal{M}_{k}\right)$ be the uncertainty space for $k=1,2, \cdots$, then the product uncertain measure $\mathcal{M}$ is an uncertain measure satisfying:

$$
\mathcal{M}\left\{\prod_{k=1}^{\infty} \Lambda_{k}\right\}=\bigwedge_{k=1}^{\infty} \mathcal{M}_{k}\left\{\Lambda_{k}\right\} .
$$

Definition 2. [25]. An uncertain variable is a measurable function $\xi$ from an uncertainty space $(\Gamma, L, \mathcal{M})$ of the set of real numbers, i.e., for any Borel set $B$ of 
real numbers, the set $\{\xi \in B\}=\{\gamma \in \Gamma \mid \xi(\gamma) \in B\}$ is an event.

An uncertainty distribution function is used to characterize an uncertain variable and defined as follows [25]:

$$
\varphi(x)=\mathcal{M}\{\xi \leq x\} \quad \forall x, x \in \mathcal{R} .
$$

Theorem 1 [44]. A function $\Phi(\mathrm{x}): \mathcal{R} \rightarrow[01]$ is an uncertainty distribution if and only if it is an increasing monotonic function, except $\Phi(x) \equiv 0$ and $\Phi(x) \equiv 1$.

Point 1. The zigzag uncertainty distribution is an uncertain variable $\xi$ shown with $\mathcal{Z}(a, b, c)$ and expressed as follows:

$$
\varphi(x)= \begin{cases}0 & x \leq a \\ \frac{(x-a)}{2(b-a)} & a \leq x \leq b \\ \frac{(x+c-2 b)}{2(c-b)} & b \leq x \leq c \\ 1 & x \geq c\end{cases}
$$

where $a, b$, and $c$ are real numbers with $a<b<c$.

Definition 3. An uncertainty distribution $\varphi$ is said to be regular if it has an inverse function $\varphi^{-1}(\alpha)$ and it is unique for each $\alpha \in(0,1)$. For instance, the uncertain distributions are given in Eq. (2).

Point 2. The inverse uncertainty distribution of zigzag uncertain variable $\mathcal{Z}(a, b, c)$ is:

$$
\Phi^{-1}(\alpha)= \begin{cases}(1-2 \alpha) a+2 \alpha b & \alpha<0.5 \\ (2-2 \alpha) b+(2 \alpha-1) c & \alpha \geq 0.5\end{cases}
$$

Definition 4 [28]. The uncertain variables $\xi_{1}, \xi_{2}, \cdots$, $\xi_{n}$ are considered to be independent if:

$$
\mathcal{M}\left\{\bigcap_{i=1}^{n}\left(\xi_{i} \in B_{i}\right)\right\}=\stackrel{\Lambda}{n=1}_{i=1}^{n} \mathcal{M}\left\{\xi_{i} \in B_{i}\right\},
$$

for any Borel set $B_{1}, B_{2}, \cdots, B_{n}$.

Definition 5 [28]. The uncertain variables $\xi_{1}, \xi_{2}, \cdots$, $\xi_{n}$ are independent if and only if:

$$
\mathcal{M}\left\{\bigcup_{i=1}^{n}\left(\xi_{i} \in B_{i}\right)\right\}=\bigvee_{i=1}^{n} \mathcal{M}\left\{\xi_{i} \in B_{i}\right\},
$$

for any Borel set $B_{1}, B_{2}, \cdots, B_{n}$.

Theorem 2 [28]. Let $\xi_{1}, \xi_{2}, \cdots, \xi_{n}$ be independent uncertain variables and $f_{1}, f_{2}, \cdots, f_{n}$ measurable functions. Then, $f_{1}\left(\xi_{1}\right), f_{2}\left(\xi_{2}\right), \cdots, f_{n}\left(\xi_{n}\right)$ are independent uncertain variables.
Theorem 3 [28]. Let $\xi_{1}, \xi_{2}, \cdots, \xi_{n}$ be variables with independent uncertainty and regular uncertainty distributions $\varphi_{1}, \varphi_{2}, \cdots, \varphi_{n}$, respectively. If $f$ is a strictly increasing function, then $\xi=f\left(\xi_{1}, \cdots, \xi_{n}\right)$ is an uncertain variable with inverse uncertain distribution $\psi^{(-1)}=f\left(\varphi_{1}^{-1}(\alpha), \varphi_{2}^{-1}(\alpha), \cdots, \varphi_{n}^{-1}(\alpha)\right)$.

Theorem 4 [28]. Let $\xi_{1}, \xi_{2}, \cdots, \xi_{n}$ be independent uncertain variables and regular uncertainty distributions $\varphi_{1}, \varphi_{2}, \cdots, \varphi_{n}$, respectively. If $f$ is strictly increasing with respect to $\xi_{1}, \xi_{2}, \cdots, \xi_{n}$ and strictly decreasing with respect to $\xi_{m+1}, \xi_{m+2}, \cdots, \xi_{m+n}$, then $\xi=f\left(\xi_{1}, \cdots, \xi_{n}\right)$ is an uncertain variable with an inverse uncertainty distribution:

$$
\begin{aligned}
\varphi^{-1}(\alpha)= & f\left(\varphi_{1}^{-1}(\alpha), \cdots, \varphi_{m}^{-1}(\alpha), \varphi_{m+1}^{-1}(1-\alpha)\right. \\
& \left.\cdots, \varphi_{n}^{-1}(1-\alpha)\right) .
\end{aligned}
$$

Definition 6 [28]. If $\xi$ be an uncertain variable, then the $\mathrm{EV}$ of $\xi$ is defined by:

$$
E[\xi]=\int_{0}^{+\infty} \mathcal{M}(\xi \geq x) d x-\int_{-\infty}^{0} \mathcal{M}(\xi \leq x) d x
$$

provided that at least one of the two integrals is finite.

The couple uncertain variable and distribution $(\xi, \varphi)$ have some related formulae explained as follows:

$$
E[\xi]=\int_{0}^{+\infty}(1-\varphi(x)) d x-\int_{-\infty}^{0} \varphi(x) d x .
$$

Point 3. The EV for variable $\xi$ with zigzag uncertain distribution is defined as fallows:

$$
E[\xi]=\frac{a+2 b+c}{4} .
$$

Theorem 5 [28]. Let $\xi_{1}, \xi_{2}, \cdots, \xi_{n}$ be independent uncertain variables with regular uncertainty distributions $\varphi_{1}, \varphi_{2}, \cdots, \varphi_{n}$, respectively. If $f$ is strictly increasing with respect to $\xi_{1}, \xi_{2}, \ldots, \xi_{n}$ and strictly decreasing with respect to $\xi_{m+1}, \xi_{m+2}, \cdots, \xi_{m+n}$, then $\xi=f\left(\xi_{1}, \cdots, \xi_{n}\right)$ has an EV:

$$
\begin{aligned}
E[\xi]= & \int_{0}^{1} f\left(\varphi_{1}^{-1}(\alpha), \cdots, \varphi_{m}^{-1}(\alpha), \varphi_{m+1}^{-1}(1-\alpha)\right. \\
& \left., \cdots, \varphi_{n}^{-1}(1-\alpha)\right) d \alpha
\end{aligned}
$$

Theorem 6 [28]. Let $\xi$ and $\eta$ be independent uncertain variables with finite $\mathrm{EV}$. Then, for any real numbers $a$ and $b$, we have:

$$
E[a \xi+b \eta]=a E[\xi]+b E[\eta]
$$


Theorem 7 [28]. Let $\xi_{1}, \xi_{2}, \cdots, \xi_{n}$ be independent uncertain variables with regular uncertainty distributions $\varphi_{1}, \varphi_{2}, \cdots, \varphi_{n}$, respectively. If the function $f\left(\xi_{1}, \cdots, \xi_{n}\right)$ is strictly increasing with respect to $\xi_{1}, \xi_{2}, \cdots, \xi_{n}$ and strictly decreasing with respect to $\xi_{m+1}, \xi_{m+2}, \cdots, \xi_{m+n}$, then: $\mathcal{M}\left\{f\left(\xi_{1}, \xi_{2}, \cdots, \xi_{n}\right) \leq\right.$ $0\} \geq \alpha$, if and only if:

$f\left(\phi_{1}^{-1}(\alpha), \cdots, \phi_{m}^{-1}(\alpha), \phi_{m+1}^{-1}(1-\alpha), \cdots, \phi_{n}^{-1}(1-\alpha)\right) \leq 0$.

Theorem 8 [28]. Let $\xi$ be an uncertain variable with regular uncertainty distribution $\Phi$. Then:

$$
E[\xi]=\int_{0}^{1} \varphi^{-1}(\alpha) d \alpha .
$$

\section{Entropy function as an uncertain variable}

The primary definition of entropy is presented in this section to specify the uncertainty of an uncertain variable.

Definition 7 [28]. Suppose that $\xi$ is an uncertain variable with uncertainty distribution $\Phi$. Then, its entropy is defined as follows:

$$
H[\xi]=\int_{-\infty}^{+\infty} S(\Phi(x)) d x,
$$

where $S(t)=-t \ln t-(1-t) \ln (1-t)$.

It is easy to verify that $S(t)$ is a symmetric function about $t=0.5$, strictly increasing in the interval $[0,0.5]$, strictly decreasing in the interval $[0.5,1]$, and reaching its unique maximum $\ln 2$ at $t=0.5$.

Point 4. Let $\xi$ be a zigzag uncertain variable $\xi \sim$ $\mathcal{Z}(a, b, c)$. Then, its entropy is:

$$
H[\xi]=\frac{c-a}{2} .
$$

Theorem 9. Let $\xi$ be an uncertain variable. Then, $H[\xi] \geq 0$ and the inequality holds if $\xi$ is essentially constant.

Theorem 10. Let $\xi$ be an uncertain variable and $c$ be a real number, then:

$$
H[\xi+c]=H[\xi] .
$$

Theorem 11 [42]. Let $\xi$ be an uncertain variable with regular uncertainty distribution $\Phi$. Then:

$$
H[\xi]=\int_{0}^{1}\left(\Phi^{-1}(\alpha) \ln \left(\frac{\alpha}{1-\alpha}\right)\right) d \alpha .
$$

Theorem 12 [42]. Let $\xi_{1}, \xi_{2}, \cdots, \xi_{n}$ be independent uncertain variables with regular uncertainty distributions $\varphi_{1}, \varphi_{2}, \cdots, \varphi_{n}$, respectively. If $f\left(x_{1}, \cdots, x_{n}\right)$ is strictly increasing with respect to $x_{1}, \cdots, x_{m}$ and strictly decreasing with respect to $x_{m+1}, \cdots, x_{m+n}$, then the uncertain variable $\xi=f\left(\xi_{1}, \cdots, \xi_{n}\right)$ has an entropy:

$$
\begin{aligned}
H[\xi]= & \int_{0}^{1} f\left(\Phi_{1}^{-1}(\alpha), \cdots, \Phi_{m}^{-1}(\alpha), \Phi_{m+1}^{-1}(1-\alpha)\right. \\
& \left.\cdots, \Phi_{n}^{-1}(1-\alpha)\right) \ln \left(\frac{\alpha}{1-\alpha}\right) d \alpha
\end{aligned}
$$

Theorem 13 [42]. Let $\xi$ and $\eta$ be independent uncertain variables. Then, for any real numbers $a$ and $b$, we have:

$$
H[a \xi+b \eta]=|a| H[\xi]+|b| H[\eta] .
$$

\section{DEA models}

Assume that there are $n$ DMUs to be evaluated, each consisting of $x_{i j}(i=1, \cdots, m)$ as input vector and $y_{r j}$ $(r=1, \cdots, s)$ as output vector. Also, $c=\left(c_{i 1}, \cdots, c_{i n}\right)$ for $(i=1, \cdots, m)$ and $p=\left(p_{r 1}, \cdots, p_{r n}\right)$ for $(r=$ $1, \cdots, s)$, respectively, represent the input costs and output prices. Assume that $D M U_{o}$ is an evaluated unit. The cost efficiency model searches for a unit which consumes the lowest cost for buying input not more than the input in the units under investigation for producing output equal to the output of the units under investigation. The cost efficiency model is defined as follows [7]:

$$
\begin{array}{ll}
c x^{*}=\min _{x, \lambda} c x=\min & \sum_{i=1}^{m} c_{i o} x_{i}, \\
\text { s.t. : } & \\
x_{i} \geq \sum_{j=1}^{n} x_{i j} \lambda_{j} & i=1, \cdots, m, \\
y_{r o} \leq \sum_{j=1}^{n} y_{r j} \lambda_{j} & r=1, \cdots, s, \\
x_{i} \geq 0 & i=1, \cdots, m, \\
\lambda_{j} \geq 0 & j=1, \cdots, n .
\end{array}
$$

Assuming an optimal solution for $\left(x^{*}, \lambda^{*}\right)$, the cost efficiency ratio is defined as:

$$
E_{c}=\frac{c x^{*}}{c x_{o}}
$$

according to Eq. (16), $0 \leq E_{c} \leq 1$.

Definition 8. $D M U_{o}$ is cost efficient if and only if $E_{c}=1$. 
In addition, the revenue efficiency model searches for a unit which gains the best revenue from selling the output greater than the output of the units under investigation and consumes the input equal to the input to the units under investigation. The revenue efficiency model is defined as follows [7]:

$$
\begin{aligned}
& p y^{*}=\max _{y, \lambda} p y=\max \sum_{r=1}^{s} p_{r o} y_{r}, \\
& \text { s.t.: } \\
& x_{i o} \geq \sum_{j=1}^{n} x_{i j} \lambda_{j} \quad i=1, \cdots, m \\
& y_{r} \leq \sum_{j=1}^{n} y_{r j} \lambda_{j} \quad r=1, \cdots, s \\
& y_{r} \geq 0 \quad r=1, \cdots, s, \\
& \lambda_{j} \geq 0 \quad j=1, \cdots, n .
\end{aligned}
$$

Assuming an optimal solution for $\left(y^{*}, \lambda^{*}\right)$, the revenue efficiency ratio is defined as:

$$
E_{R}=\frac{p y_{o}}{p y^{*}},
$$

according to Eq. (18), $0 \leq E_{R} \leq 1$.

Definition 9. $D M U_{o}$ is revenue-efficient if and only if $E_{R}=1$.

\section{Uncertain DEA model}

The allocation model requires input and output equipped with precise data. Nevertheless, in realworld situations, input and output may be unstable and complicated and, therefore, cannot be measured in an accurate manner. This conflict results in the investigation of uncertain DEA models. Decisionmakers in real-word situations make their decisions in an indeterminate state. To model indeterminacy, there exist two mathematical systems, namely the probability theory [45] and the uncertainty theory [25]. If there exists frequency in the phenomena, the probability theory is employed; otherwise, the uncertain theory can be a powerful technique for resolving a problem with no sample using the personal belief degree. For this purpose, skilled consultants and experts should be invited to measure the belief degree. Belief degreebased uncertainty is useful for cases in which there is no historical information on an uncertain event. For example, both costs and prices may be unstable and complex in the stock market. Therefore, uncertain DEA models should be used for discovering the efficiency of two people with two different stock portfolios in terms of cost and revenue.

Through this approach, we aim to introduce an allocation model, referred to as the uncertain allocation model, with uncertain input and output. First, the new symbols and notation are presented as follows:

- $\tilde{x}_{k}=\left(\tilde{x}_{1 k}, \tilde{x}_{2 k}, \cdots, \tilde{x}_{m k}\right):$ The uncertain input vector of $D M U_{k}, k=1,2, \cdots, n$;

- $\varphi_{i k}(x)$ : The uncertainty distribution of $\tilde{x}_{i k}, k=$ $1,2, \cdots, n, i=1,2, \cdots, m$;

- $\tilde{y}_{k}=\left(\tilde{y}_{1 k}, \cdots, \tilde{y}_{r k}\right)$ : The uncertain output vector of $D M U_{k}, k=1,2, \cdots, n$;

- $\psi_{r k}(x)$ : The uncertainty distribution of $\tilde{y}_{r k}, k=$ $1,2, \cdots, n, r=1,2, \cdots, s$;

- $\alpha$ : A predetermined confidence level;

- $\mathcal{M}$ : The uncertainty measure expressed in Section 2.

Now, suppose that the input $\operatorname{costs} c_{i j}$ and the input vectors $x_{i j}$, for $(i=1, \cdots, m),(j=1, \cdots, n)$ as well as the output vector $y_{r j}(r=1, \cdots, s)$ in Model (15) are uncertain variables represented by $\tilde{c}_{i j}, \tilde{x}_{i j}$, and $\tilde{y}_{r j}$, respectively. Accordingly, the uncertain cost efficiency model can be rewritten as follows:

$$
\begin{aligned}
& \tilde{c} x^{*}=\min _{x, \lambda} \tilde{c} x=\min \sum_{i=1}^{m} \tilde{c}_{i o} x_{i}, \\
& \text { s.t.: } \\
& x_{i} \geq \sum_{j=1}^{n} \tilde{x}_{i j} \lambda_{j} \quad i=1, \cdots, m, \\
& \tilde{y}_{r o} \leq \sum_{j=1}^{n} \tilde{y}_{r j} \lambda_{j} \quad r=1, \cdots, s, \\
& \lambda_{j} \geq 0, \quad x_{i} \geq 0 \quad j=1, \cdots, n, \quad i=1, \cdots, m .
\end{aligned}
$$

Also, suppose that the output price $p_{r j}$, the output vectors $y_{r j}$ for $(r=1, \cdots, s),(j=1, \cdots, n)$, and the input vector $x_{i j}(i=1, \cdots, m)$ in Model (17) are uncertain variables represented by $\tilde{p}_{r j}, \tilde{y}_{r j}$, and $\tilde{x}_{i j}$, respectively. Accordingly, the uncertain cost efficiency model can be rewritten as follows:

$$
\begin{aligned}
& \tilde{p} y^{*}=\max _{y, \lambda} \tilde{p} y=\max \sum_{r=1}^{s} \tilde{p}_{r o} y_{r}, \\
& \text { s.t.: } \\
& \tilde{x}_{i o} \geq \sum_{j=1}^{n} \tilde{x}_{i j} \lambda_{j} \\
& y_{r} \leq \sum_{j=1}^{n} \tilde{y}_{r j} \lambda_{j} \\
& \lambda_{j} \geq 0, \quad y_{r} \geq 0 \quad j=1, \cdots, m,
\end{aligned}
$$




\section{Crisp equivalents of the model}

To deal with the uncertainty problem, two different methods are presented to convert it into a crisp model, namely (1) the EV and (2) the EVCC methods.

\section{1. $E V$ method}

In this section, the uncertain cost model is transformed to a crisp model using the EV method.

\subsubsection{Cost optimization model}

The uncertain cost efficiency (Model (19)) is introduced as follows using the EV method:

$$
\begin{aligned}
& \theta=E\left[\tilde{c} x^{*}\right]=\min _{x, \lambda} E[\tilde{c} x]=\min E\left[\sum_{i=1}^{m} \tilde{c}_{i o} x_{i}\right], \\
& \text { s.t.: } \\
& E\left[\sum_{j=1}^{n} \tilde{x}_{i j} \lambda_{j}-x_{i}\right] \leq 0 \quad i=1, \cdots, m, \\
& E\left[\begin{array}{l}
\left.\tilde{y}_{r o}-\sum_{j=1}^{n} \tilde{y}_{r j} \lambda_{j}\right] \leq 0 \\
\lambda_{j} \geq 0, \quad x_{i} \geq 0 \quad j=1, \cdots, n,
\end{array}\right]=1, \cdots, s,
\end{aligned}
$$

Definition 10 (efficiency). In Model (21), $D M U_{o}$ is efficient if and only if $\theta^{*}=1$, where $\theta^{*}$ is the optimal value of Model (21).

Theorem 14. Assume that the input costs $\tilde{c}_{i 1}, \tilde{c}_{i 2}$, $\cdots, \tilde{c}_{i n}$, the input $\tilde{x}_{i 1}, \tilde{x}_{i 2}, \cdots, \tilde{x}_{i n}$, and the output $\tilde{y}_{r 1}, \tilde{y}_{r 2}, \cdots, \tilde{y}_{r n}$ are independent uncertain variables with uncertainty distributions of $\varphi_{i 1}, \varphi_{i 2}, \cdots, \varphi_{i n}$; $\psi_{i 1}, \psi_{i 2}, \cdots, \psi_{i n} ;$ and $\chi_{r 1}, \chi_{r 2}, \cdots, \chi_{r n}$, respectively, where $i=1,2, \cdots, m$ and $r=1, \cdots, s$. Then, the uncertain programming Model (21) will be equivalent to the following model:

$$
\min \sum_{i=1}^{m} x_{i} \int_{0}^{1} \Phi_{i o}^{-1}(\alpha) d \alpha,
$$

s.t.:

$\sum_{j=1}^{n} \lambda_{j} \int_{0}^{1} \psi_{i j}^{-1}(\alpha) d \alpha-x_{i} \leq 0 \quad i=1, \cdots, m$

$\int_{0}^{1} \chi_{r_{o}}^{-1}(\alpha) d \alpha-\sum_{j=1}^{n} \lambda_{j} \int_{0}^{1} \chi_{r j}^{-1}(1-\alpha) d \alpha \leq 0$

$$
r=1, \cdots, s,
$$

$$
\lambda_{j} \geq 0, \quad x_{i} \geq 0 \quad j=1, \cdots, n, \quad i=1, \cdots, m .
$$

Proof. According to Theorem 6, objective function in (21) is rewritten as follows:

$$
E\left[\sum_{i=1}^{m} \tilde{c}_{i o} x_{i}\right]=\sum_{i=1}^{m} E\left[\tilde{c}_{i o} x_{i}\right]=\sum_{i=1}^{m} x_{i} E\left[\tilde{c}_{i o}\right]
$$

The function $\sum_{i=1}^{m} x_{i} E\left[\tilde{c}_{i o}\right]$ is strictly increasing with respect to $\tilde{c}_{i o}$ for each $i$. According to Theorem 8 , we have:

$$
\sum_{i=1}^{m} x_{i} E\left[\tilde{c}_{i o}\right]=\sum_{i=1}^{m} x_{i} \int_{0}^{1} \Phi_{i o}^{-1}(\alpha) d \alpha
$$

As a result, the objective function is proven. Now, according to Theorem 8:

$$
E[a \xi]=\int_{0}^{1} a \Phi^{-1}(\alpha) d \alpha=a \int_{0}^{1} \Phi^{-1}(\alpha) d \alpha=a E[\xi]
$$

Let us prove the constraint as follows:

According to Theorem 6 , the first constraint of Model (21) is rewritten as follows:

$$
\begin{aligned}
& E\left[\sum_{j=1}^{n} \tilde{x}_{i j} \lambda_{j}-x_{i}\right]=E\left[\sum_{j=1}^{n} \tilde{x}_{i j} \lambda_{j}\right]-x_{i} \\
& =\sum_{j=1}^{n} \lambda_{j} E\left[\tilde{x}_{i j}\right]-x_{i}=\sum_{j=1}^{n} \lambda_{j} \int_{0}^{1} \psi_{i j}^{-1}(\alpha) d \alpha-x_{i} .
\end{aligned}
$$

The function $\tilde{y}_{r o}-\sum_{j=1}^{n} \tilde{y}_{r j} \lambda_{j}$ is strictly increasing with respect to $\tilde{y}_{r o}$ and strictly decreasing with respect to $\tilde{y}_{r j}$ for each $r, r=1, \cdots, s$ and $j, j=1, \cdots, n$. According to Theorems 6 and 8 , we have:

$$
\begin{gathered}
E\left[\tilde{y}_{r o}-\sum_{j=1}^{n} \tilde{y}_{r j} \lambda_{j}\right]=E\left[\tilde{y}_{r o}\right]-E\left[\sum_{j=1}^{n} \tilde{y}_{r j} \lambda_{j}\right] \\
=\int_{0}^{1} \chi_{r o}^{-1}(\alpha) d \alpha-\sum_{j=1}^{n} \lambda_{j} \int_{0}^{1} \chi_{r j}^{-1}(1-\alpha) d \alpha .
\end{gathered}
$$

The theorem is therefore proven.

\subsubsection{Revenue optimization model}

Using the EV method explained in the previous section, the uncertain revenue (Model (20)) is introduced as follows: 


$$
\begin{aligned}
& \theta=E\left[\tilde{p} y^{*}\right]=\max _{y, \lambda} E[\tilde{p} y]=\max _{y, \lambda} E\left[\sum_{r=1}^{s} \tilde{p}_{r o} y_{r}\right], \\
& \text { s.t.: } \\
& E\left[\sum_{j=1}^{n} \tilde{x}_{i j} \lambda_{j}-\tilde{x}_{i o}\right] \leq 0 \\
& E\left[\begin{array}{c}
\left.y_{r}-\sum_{j=1}^{n} \tilde{y}_{r j} \lambda_{j}\right] \leq 0 \\
\quad
\end{array}\right]=1, \cdots, m, \\
& \lambda_{j} \geq 0, \quad y_{r} \geq 0 \quad j=1, \cdots, n, \quad r=1, \cdots, s .
\end{aligned}
$$

Definition 11 (efficiency). In Model (23), $D M U_{o}$ is efficient if and only if $\theta^{*}=1$, where $\theta^{*}$ is the optimal value of Model (23).

Theorem 15. Assume that the output prices $\tilde{p}_{r 1}, \tilde{p}_{r 2}$, $\cdots, \tilde{p}_{r n}$, the input $\tilde{x}_{i 1}, \tilde{x}_{i 2}, \cdots, \tilde{x}_{i n}$, and the output $\tilde{y}_{r 1}, \tilde{y}_{r 2}, \cdots, \tilde{y}_{r n}$ are independent uncertain variables with uncertainty distributions $\tau_{r 1}, \tau_{r 2}, \cdots, \tau_{r n}$; $\psi_{i 1}, \psi_{i 2}, \cdots, \psi_{i n}$; and $\chi_{r 1}, \chi_{r 2}, \cdots, \chi_{r n}$, respectively, where $i=1,2, \cdots, m$ and $r=1, \cdots, s$. Then, the uncertain programming (Model (23)) will be equivalent to the following model:

$$
\max \sum_{r=1}^{s} y_{r} \int_{0}^{1} \tau_{r o}^{-1}(\alpha) d \alpha
$$

s.t.:

$$
\begin{aligned}
& \sum_{j=1}^{n} \lambda_{j} \int_{0}^{1} \Psi_{i j}^{-1}(\alpha)-\int_{0}^{1} \Psi_{i o}^{-1}(1-\alpha) d \alpha \leq 0 \\
& i=1,2, \cdots, m, \\
& y_{r}-\sum_{j=1}^{n} \lambda_{j} \int_{0}^{1} \chi_{r j}^{-1}(1-\alpha) d \alpha \quad r=1, \cdots, s, \\
& \lambda_{j} \geq 0, \quad y_{r} \geq 0, \quad j=1, \cdots, n, \quad r=1, \cdots, s .
\end{aligned}
$$

Proof. According to Theorem 6, the objective function in Model (23) is rewritten as follows:

$$
E\left[\sum_{r=1}^{s} \tilde{p}_{r o} y_{r}\right]=\sum_{r=1}^{s} E\left[\tilde{p}_{r o} y_{r}\right]=\sum_{r=1}^{s} y_{r} E\left[\tilde{p}_{r o}\right] .
$$

The function $\sum_{r=1}^{s} y_{r} E\left[\tilde{p}_{r o}\right]$ is strictly increasing with respect to $\tilde{p}_{r o}$ for each $r$. According to Theorem 8, we have:

$$
\sum_{r=1}^{s} y_{r} E\left[\tilde{p}_{r o}\right]=\sum_{r=1}^{s} y_{r} \int_{0}^{1} \tau_{i o}^{-1}(\alpha) d \alpha .
$$

Thus, the objective function is proven. Now, according to Theorem 8:

$$
E[a \xi]=\int_{0}^{1} a \Psi^{-1}(\alpha) d \alpha=a \int_{0}^{1} \Psi^{-1}(\alpha) d \alpha=a E[\xi] .
$$

Let us prove the constraint as follows. According to Theorem 6, the first constraint of Model (23) is rewritten as follows:

$$
\begin{aligned}
& E\left[y_{r}-\sum_{j=1}^{n} \tilde{y}_{r j} \lambda_{j}\right]=y_{r}-E\left[\sum_{j=1}^{n} \tilde{y}_{r j} \lambda_{j}\right] \\
& \quad=y_{r}-\sum_{j=1}^{n} E\left[\tilde{y}_{r j}\right] \lambda_{j} .
\end{aligned}
$$

The function $y_{r}-\sum_{j=1}^{n} E\left[\tilde{y}_{r j}\right] \lambda_{j}$ is strictly increasing with respect to $-\tilde{y}_{r j}$ for each $r=1, \cdots, s$ and $j=1, \cdots, n$. According to Theorem 8, we have:

$$
\begin{aligned}
& y_{r}-\sum_{j=1}^{n} E\left[\tilde{y}_{r j}\right] \lambda_{j}=y_{r}-\sum_{j=1}^{n} \lambda_{j} \int_{0}^{1} \chi_{r j}^{-1}(1-\alpha) d \alpha \\
& \forall r, r=1, \cdots, s .
\end{aligned}
$$

The function $\sum_{j=1}^{n} \tilde{x}_{i j} \lambda_{j}-\tilde{x}_{i o}$ is strictly increasing with respect to $\tilde{x}_{i j}$ and strictly decreasing with respect to $-\tilde{x}_{i o}$ for each $i=1, \cdots, m$ and $j=1, \cdots, n$. According to Theorems 6 and 8 , we have:

$$
\begin{gathered}
E\left[\sum_{j=1}^{n} \tilde{x}_{i j} \lambda_{j}-\tilde{x}_{i o}\right]=\sum_{j=1}^{n} \lambda_{j} E\left[\tilde{x}_{i j}\right]-E\left[\tilde{x}_{i o}\right] \\
=\sum_{j=1}^{n} \lambda_{j} \int_{0}^{1} \Psi_{i j}^{-1}(\alpha) d \alpha-\int_{0}^{1} \Psi_{i o}^{-1}(1-\alpha) d \alpha \\
i=1, \cdots, m
\end{gathered}
$$

The theorem is therefore proven.

\section{2. $E V C C$ method}

Using the EVCC method, the uncertain cost and revenue models are converted into crisp models and the new crisp models are solved with the help of specific software.

\subsubsection{Cost optimization model}

The uncertain cost (Model (19)) is converted to a crisp model using the EVCC method as follows: 


$$
\begin{aligned}
& E\left[\tilde{c} x^{*}\right]=\min _{x, \lambda} E[\tilde{c} x]=\min E\left[\sum_{i=1}^{m} \tilde{c}_{i o} x_{i}\right], \\
& \text { s.t.: } \\
& \mathcal{M}\left\{\sum_{j=1}^{n} \tilde{x}_{i j} \lambda_{j}-x_{i} \leq 0\right\} \geq \alpha \quad i=1,2, \cdots, m, \\
& \mathcal{M}\left\{\tilde{y}_{r o}-\sum_{j=1}^{n} \tilde{y}_{r j} \lambda_{j} \leq 0\right\} \geq \alpha \quad r=1, \cdots, s,
\end{aligned}
$$$$
\lambda_{j} \geq 0, \quad x_{i} \geq 0, \quad j=1, \cdots, n, \quad i=1, \cdots, m .
$$

Definition 12. A vector $\left(x_{i}, \lambda_{j}\right) \geq 0$ where $j=$ $1, \ldots, n, i=1, \ldots, m$ is called a feasible solution to the uncertain programming (Model (25)) if:

$$
\begin{aligned}
& \mathcal{M}\left\{\sum_{j=1}^{n} \tilde{x}_{i j} \lambda_{j}-x_{i} \leq 0\right\} \geq \alpha \quad i=1, \cdots, m . \\
& \mathcal{M}\left\{\tilde{y}_{r o}-\sum_{j=1}^{n} \tilde{y}_{r j} \lambda_{j} \leq 0\right\} \geq \alpha \quad r=1, \cdots, s .
\end{aligned}
$$

Definition 13. A feasible solution is called an expected optimal solution to the uncertain programming (Model (25)) if:

$$
\begin{aligned}
& E\left[\sum_{i=1}^{m} \tilde{c}_{i o} x_{i}^{*}\right] \leq E\left[\sum_{i=1}^{m} \tilde{c}_{i o} x_{i}\right] \\
& i=1,2, \cdots, m, \quad j=1,2, \cdots, n,
\end{aligned}
$$

for any solution $\left(x_{i}, \lambda_{j}\right)$.

Definition 14. A larger optimal objective value in Model (25) means a more efficient $D M U_{o}$.

Theorem 16. Assume that the input costs $\tilde{c}_{i 1}, \tilde{c}_{i 2}$, $\cdots, \tilde{c}_{i n}$, the input $\tilde{x}_{i 1}, \tilde{x}_{i 2}, \cdots, \tilde{x}_{i n}$, and the output $\tilde{y}_{r 1}, \tilde{y}_{r 2}, \cdots, \tilde{y}_{r n}$, are independent uncertain variables with uncertainty distributions of $\varphi_{i 1}, \varphi_{i 2}, \cdots, \varphi_{i n}$; $\psi_{i 1}, \psi_{i 2}, \cdots, \psi_{i n}$; and $\chi_{r 1}, \chi_{r 2}, \cdots, \chi_{r n}$, respectively, where $i=1,2, \cdots, m$ and $r=1, \cdots, s$. Then, the uncertain programming (Model (25)) will be equivalent to the following model:

$$
\min \sum_{i=1}^{m} x_{i} \int_{0}^{1} \Phi_{i o}^{-1}(\alpha) d \alpha
$$

s.t.:

$$
\begin{array}{ll}
\sum_{j=1}^{n} \psi_{i j}^{-1}(\alpha) \lambda_{j}-x_{i} \leq 0 & i=1,2, \cdots, m, \\
\chi_{r o}^{-1}(\alpha)-\sum_{j=1}^{n} \chi_{r j}^{-1}(1-\alpha) \lambda_{j} \leq 0 & r=1, \cdots, s, \\
\lambda_{j} \geq 0, \quad x_{i} \geq 0 \quad i=1,2, \cdots, m, & j=1,2, \cdots, n .
\end{array}
$$

Proof. Equivalency of the objective function was proven in the proof of Theorem 15. To prove the equivalency of the constraints, the function $\sum_{j=1}^{n} \tilde{x}_{i j} \lambda_{j}-x_{i}$ is strictly increasing with respect to $\tilde{x}_{i j}$ for each $i=$ $1, \cdots, m$ and $j=1, \cdots, n$. According to Theorem 7:

$$
\begin{gathered}
\mathcal{M}\left\{\sum_{j=1}^{n} \tilde{x}_{i j} \lambda_{j}-x_{i} \leq 0\right\} \geq \alpha \Leftrightarrow \\
\mathcal{M}\left\{\sum_{j=1}^{n} \tilde{x}_{i j} \lambda_{j}-x_{i} \leq 0\right\} \geq \alpha \Leftrightarrow \sum_{j=1}^{n} \psi_{i j}^{-1}(\alpha) \lambda_{j}-x_{i} \leq 0, \\
\forall j, \quad j=1, \cdots, n, \quad i=1, \cdots, m .
\end{gathered}
$$

Also, the function $\tilde{y}_{r o}-\sum_{j=1}^{n} \tilde{y}_{r j} \lambda_{j}$ is strictly increasing with respect to $\tilde{y}_{r o}$ and strictly decreasing with respect to $\tilde{y}_{r j}$ for each $r=1, \cdots, s$ and $j=1, \cdots, n$. According to Theorem 7:

$$
\begin{gathered}
\mathcal{M}\left\{\tilde{y}_{r o}-\sum_{j=1}^{n} \tilde{y}_{r j} \lambda_{j} \leq 0\right\} \geq \alpha \Leftrightarrow \chi_{r o}^{-1}(\alpha) \\
-\sum_{j=1}^{n} \chi_{r j}^{-1}(1-\alpha) \lambda_{j} \leq 0 \\
j=1, \cdots, n, \quad r=1, \cdots, s .
\end{gathered}
$$

Therefore, the theorem is proven.

\subsubsection{Revenue optimization model}

The uncertain revenue (Model (20)) is converted to a crisp model using the EVCC method:

$$
E\left[\tilde{p} y^{*}\right]=\max _{y, \lambda} E[\tilde{p} y]=\max E\left[\sum_{r=1}^{s} \tilde{p}_{r o} y_{r}\right]
$$

s.t.:

$$
\begin{aligned}
& \mathcal{M}\left\{\sum_{j=1}^{n} \tilde{x}_{i j} \lambda_{j}-\tilde{x}_{i o} \leq 0\right\} \geq \alpha \quad i=1,2, \cdots, m, \\
& \mathcal{M}\left\{y_{r}-\sum_{j=1}^{n} \tilde{y}_{r j} \lambda_{j} \leq 0\right\} \geq \alpha, \quad r=1, \cdots, s,
\end{aligned}
$$


$\lambda_{j} \geq 0, \quad y_{r} \geq 0 \quad j=1, \cdots, n, \quad r=1, \cdots, s$.

Definition 15. A vector $\left(y_{r}, \lambda_{j}\right) \geq 0$ where $j=$ $1, \ldots, n, r=1, \ldots, s$, is called a feasible solution to the uncertain programming (Model (28)) if:

$$
\begin{aligned}
& \mathcal{M}\left\{\sum_{j=1}^{n} \tilde{x}_{i j} \lambda_{j}-\tilde{x}_{i o} \leq 0\right\} \geq \alpha \quad i=1,2, \cdots, m, \\
& \mathcal{M}\left\{y_{r}-\sum_{j=1}^{n} \tilde{y}_{r j} \lambda_{j} \leq 0\right\} \geq \alpha \quad r=1, \cdots, s .
\end{aligned}
$$

Definition 16. A feasible solution $\left(y_{r}^{*}, \lambda_{j}^{*}\right)$ is called an expected optimal solution to the uncertain programming (Model (28)) if:

$$
\begin{gathered}
E\left[\sum_{r=1}^{s} \tilde{p}_{r o} y_{r}^{*}\right] \geq E\left[\sum_{r=1}^{s} \tilde{p}_{r o} y_{r}\right] \\
j=1, \cdots, n, \quad r=1, \cdots, s,
\end{gathered}
$$

for any solution $\left(y_{r}, \lambda_{j}\right)$.

Definition 17. A larger optimal objective value in Model (28) means a more efficient $D M U_{o}$.

Theorem 17. Assume that the output prices $\tilde{p}_{r 1}, \tilde{p}_{r 2}$, $\cdots, \tilde{p}_{r n}$, the input $\tilde{x}_{i 1}, \tilde{x}_{i 2}, \cdots, \tilde{x}_{i n}$, and the output $\tilde{y}_{r 1}, \tilde{y}_{r 2}, \cdots, \tilde{y}_{r n}$ are independent uncertain variables with uncertainty distributions of $\tau_{r 1}, \tau_{r 2}, \cdots, \tau_{r n}$; $\psi_{i 1}, \psi_{i 2}, \cdots, \psi_{i n}$; and $\chi_{r 1}, \chi_{r 2}, \cdots, \chi_{r n}$, respectively, where $i=1,2, \cdots, m$ and $r=1, \cdots, s$. Then, the uncertain programming (Model (28)) will be equivalent to the following model:

$$
\max \sum_{r=1}^{s} y_{r} \int_{0}^{1} \tau_{r o}^{-1}(\alpha) d \alpha
$$

s.t.:

$$
\begin{array}{ll}
\sum_{j=1}^{n} \lambda_{j} \psi_{i j}^{-1}(\alpha)-\psi_{i o}^{-1}(1-\alpha) \leq 0 & i=1,2, \cdots, m, \\
y_{r}-\sum_{j=1}^{n} \chi_{r j}^{-1}(1-\alpha) \lambda_{j} \leq 0 & r=1, \cdots, s, \\
\lambda_{j} \geq 0, \quad y_{r} \geq 0 \quad j=1, \cdots, n, & r=1, \cdots, s .
\end{array}
$$

Proof. Equivalency of objective function was proven in the proof of Theorem 15. To prove the equivalency of the constraints, the function $\sum_{j=1}^{n} \tilde{x}_{i j} \lambda_{j}-\tilde{x}_{i o}$ is strictly increasing with respect to $\tilde{x}_{i j}$ and strictly decreasing with respect to $-\tilde{x}_{i o}$ for each $i=1, \cdots, m$, and $j=$ $1, \cdots, n$. According to Theorem 7 , we have:

$$
\begin{aligned}
& \mathcal{M}\left\{\sum_{j=1}^{n} \tilde{x}_{i j} \lambda_{j}-\tilde{x}_{i o} \leq 0\right\} \geq \alpha \Leftrightarrow \\
& \mathcal{M}\left\{\sum_{j=1}^{n} \tilde{x}_{i j} \lambda_{j}-\tilde{x}_{i o} \leq 0\right\} \geq \alpha \Leftrightarrow \sum_{j=1}^{n} \lambda_{j} \psi_{i j}^{-1}(\alpha) \\
& -\psi_{i o}^{-1}(1-\alpha) \leq 0 \quad j=1, \cdots, n, \quad i=1, \cdots, m .
\end{aligned}
$$

Also, the function $y_{r}-\sum_{j=1}^{n} \tilde{y}_{r j} \lambda_{j}$ is strictly decreasing with respect to $-\tilde{y}_{r j}$ for each $r=1, \cdots, s$, and $j=$ $1, \cdots, n$. According to Theorem 7 , we have:

$$
\begin{gathered}
\mathcal{M}\left\{y_{r}-\sum_{j=1}^{n} \tilde{y}_{r j} \lambda_{j} \leq 0\right\} \geq \alpha \Leftrightarrow y_{r} \\
-\sum_{j=1}^{n} \chi_{r j}^{-1}(1-\alpha) \lambda_{j} \leq 0 \\
j=1, \cdots, n, \quad r=1, \cdots, s .
\end{gathered}
$$

Proof is completed.

\section{Entropy-based allocation models with uncertain variables}

In the literature on uncertain DEA, there is no study on the use of entropy in models with uncertain data. Entropy is employed to provide a quantitative measure for the degree of uncertainty. On the basis of the Shannon and Weaver [46] entropy, Liu [47] introduced the concept of entropy for the first time to determine the uncertainty of uncertain variables resulting from information deficiency. Chen and Dai [41] and Dai and Chen [42] investigated the principle of maximum entropy of uncertainty distribution for uncertain variables. They presented the entropy of a function for uncertain variables.

Considering the maximum entropy in allocation models help us achieve the highest revenue and the lowest cost with the maximum dispersal measure. This model will result in the efficiency of units, which are considered more dispersed in all processes. Moreover, it will enhance the power of managerial decisions.

Now, Model (12) is rewritten as a second objective function using Models (22), (24), (27), and (30) as follows. By utilizing Theorems 12 and 13 as the objective function of Model (22), we define the following uncertain entropy function. 
Lemma 1. Suppose $\tilde{c}_{i 1}, \tilde{c}_{i 2}, \cdots, \tilde{c}_{i n}$ is an uncertain variable with regular uncertainty distribution $\varphi_{i 1}, \varphi_{i 2}, \cdots, \varphi_{\text {in }}$ for $(i=1, \cdots, m)$. If $f: \mathcal{R}^{n} \rightarrow \mathcal{R}$ is a strictly increasing function with respect to $x_{i}$, $i=1, \cdots, m$, then the uncertain function $f(x, \tilde{c})$ has an entropy:

$$
H(x, \tilde{c})=\sum_{i=1}^{m} x_{i} \int_{0}^{1} \varphi_{i o}^{-1}(\alpha) \ln \left(\frac{\alpha}{1-\alpha}\right) d \alpha .
$$

Proof. Since $\tilde{c}_{i o}$ has a regular uncertain distribution $\varphi_{i o}$, we obtain:

$$
H(x, \tilde{c})=\sum_{i=1}^{m} x_{i} \int_{-\infty}^{+\infty} f\left(\varphi_{i o}(x)\right) d x .
$$

From Theorem 13, the following equality can be rewritten:

$$
\begin{aligned}
H(x, \tilde{c})= & \sum_{i=1}^{m} x_{i} \int_{-\infty}^{0} \int_{0}^{\varphi_{i o}(x)} f^{\prime}(\alpha) d \alpha d x \\
& +\sum_{i=1}^{m} x_{i} \int_{-\infty}^{0} \int_{\varphi_{i o}(x)}^{+\infty}-f^{\prime}(\alpha) d \alpha d x
\end{aligned}
$$

where:

$$
\begin{aligned}
f^{\prime}(\alpha) & =(-\alpha \ln \alpha-(1-\alpha) \ln (1-\alpha))^{\prime} \\
& =-\ln \left(\frac{\alpha}{1-\alpha}\right) .
\end{aligned}
$$

By applying the Fubini theorem [48] to the above function, we obtain:

$$
\begin{aligned}
H(x, \tilde{c})= & \sum_{i=1}^{m} x_{i} \int_{-\infty}^{0} \int_{\varphi_{i o}^{-1}(x)}^{0} f^{\prime}(\alpha) d \alpha d x \\
& +\sum_{i=1}^{m} x_{i} \int_{\varphi_{i o}(x)}^{1} \int_{0}^{\varphi_{i o}(x)}-f^{\prime}(\alpha) d \alpha d x \\
= & \sum_{i=1}^{m} x_{i} \int_{0}^{1} \varphi_{i o}^{-1}(\alpha) f^{\prime}(\alpha) d \alpha \\
= & \sum_{i=1}^{m} x_{i} \int_{0}^{1} \varphi_{i o}^{-1}(\alpha) \ln \left(\frac{\alpha}{1-\alpha}\right) d \alpha .
\end{aligned}
$$

Lemma 2. Suppose $\tilde{p}_{r 1}, \tilde{p}_{r 2}, \cdots, \tilde{p}_{r n}$ is an uncertain variable with regular uncertainty distribution $\tau_{r 1}, \tau_{r 2}, \cdots, \tau_{r n}$ for $r=1, \cdots, s$. If $f: R^{n} \rightarrow R$ is a strictly increasing function with respect to $y_{r}$, $r=1, \cdots, s$, then the uncertain function $f(y, \tilde{p})$ has an entropy:

$$
H(y, \tilde{p})=\sum_{r=1}^{s} y_{r} \int_{0}^{1} \tau_{r o}^{-1}(\alpha) \ln \left(\frac{\alpha}{1-\alpha}\right) d \alpha .
$$

Proof. Since $\tilde{p}_{\text {ro }}$ has a regular uncertain distribution $\tau_{\text {ro }}$, we obtain:

$$
H(y, \tilde{p})=\sum_{r=1}^{s} y_{r} \int_{-\infty}^{+\infty} f\left(\tau_{r o}(x)\right) d x .
$$

From Theorem 13, this equality can be rewritten as:

$$
\begin{aligned}
H(y, \tilde{p})= & \sum_{r=1}^{s} y_{r} \int_{-\infty}^{0} \int_{0}^{\tau_{r o}(x)} f^{\prime}(\alpha) d \alpha d x \\
& +\sum_{r=1}^{s} y_{r} \int_{-\infty}^{0} \int_{\tau_{r o}(x)}^{\infty}-f^{\prime}(\alpha) d \alpha d x,
\end{aligned}
$$

where:

$$
f^{\prime}(\alpha)=(-\alpha \ln \alpha-(1-\alpha) \ln (1-\alpha))^{\prime}=-\ln \frac{\alpha}{1-\alpha} .
$$

By applying the Fubini theorem [48] to the above function, we obtain:

$$
\begin{aligned}
H(y, \tilde{p})= & \sum_{r=1}^{s} y_{r} \int_{-\infty}^{0} \int_{\tau_{r_{o}}^{-1}(x)}^{0} f^{\prime}(\alpha) d \alpha d x \\
& +\sum_{r=1}^{s} y_{r} \int_{\tau_{r o}(x)}^{1} \int_{0}^{\tau_{r o}(x)}-f^{\prime}(\alpha) d \alpha d x \\
= & \sum_{r=1}^{s} y_{r} \int_{0}^{1} \tau_{r o}^{-1}(\alpha) f^{\prime}(\alpha) d \alpha \\
= & \sum_{r=1}^{s} y_{r} \int_{0}^{1} \tau_{r o}^{-1}(\alpha) \ln \left(\frac{\alpha}{1-\alpha}\right) d \alpha .
\end{aligned}
$$

According to above discussions, the allocative efficiency models are presented below. First, the new multiobjective cost efficiency model consisting of an uncertain cost model and an uncertain entropy function is explained.

Note 1. All multi-objective problems are solved by the weighting method. Accordingly, the multiobjective problems are converted into single-objective ones. The coefficients $w_{1}$ and $w_{2}$ are considered for improving the flexibility of our model to be used by decision makers for determining the importance of objective functions as $w_{1}+w_{2}=1$. The values of these functions are determined before solving the problems by the decision makers. Equal weights $\left(w_{1}=w_{2}\right)$ mean equal attention of decision makers to both functions; the uncertain multi-objective cost efficiency model is introduced as follows using the EVCC method. The EVCC method is used for converting the new model to a crisp model: 


$$
\begin{aligned}
& Z_{1}=\min \sum_{i=1}^{m} x_{i} \int_{0}^{1} \Phi_{i o}^{-1}(\alpha) d \alpha \\
& Z_{2}=\max \sum_{i=1}^{m} x_{i} \int_{0}^{1} \Phi_{i o}^{-1}(\alpha) \ln \frac{\alpha}{1-\alpha} d \alpha
\end{aligned}
$$

s.t.:

$$
\begin{array}{ll}
\sum_{j=1}^{n} \psi_{i j}^{-1}(\alpha) \lambda_{j}-x_{i} \leq 0 & i=1, \cdots, m, \\
\chi_{r o}^{-1}(\alpha)-\sum_{j=1}^{n} \chi_{r j}^{-1}(1-\alpha) \lambda_{j} \leq 0, & r=1, \cdots, s, \\
\lambda_{j} \geq 0, \quad x_{i} \geq 0 \quad j=1, \cdots, n, & i=1, \cdots, m .
\end{array}
$$

Using the weighting method explained previously, the uncertain multi-objective (Model (33)) is rewritten as follows:

$$
\begin{array}{ll}
\min \left(w_{1} Z_{1}-w_{2} Z_{2}\right), & \\
\text { s.t.: } & i=1, \cdots, m, \\
\sum_{j=1}^{n} \psi_{i j}^{-1}(\alpha) \lambda_{j}-x_{i} \leq 0 & r=1, \cdots, s, \\
\chi_{r o}^{-1}(\alpha)-\sum_{j=1}^{n} \chi_{r j}^{-1}(1-\alpha) \lambda_{j} \leq 0 & \\
\lambda_{j} \geq 0, \quad x_{i} \geq 0 \quad j=1, \cdots, n, & i=1, \cdots, m .
\end{array}
$$

Definition 18. The greater the optimal objective value in Model (34) is, the more efficient is the $D M U_{o}$.

The new multi-objective revenue efficiency model consisting of an uncertain revenue model as the first objective function and an uncertain entropy as the second objective function is developed here. The uncertain multi-objective revenue efficiency model is introduced using the EVCC method as follows. Again, EVCC method is used for converting the uncertain form to the crisp form.

$$
\begin{aligned}
& Z_{1}=\max \sum_{r=1}^{s} y_{r} \int_{0}^{1} \Psi_{r o}^{-1}(\alpha) d \alpha, \\
& Z_{2}=\max \sum_{r=1}^{s} y_{r} \int_{0}^{1} \Psi_{r o}^{-1}(\alpha) \ln \frac{\alpha}{1-\alpha} d \alpha, \\
& \text { s.t.: } \\
& \sum_{j=1}^{n} \lambda_{j} \psi_{i j}^{-1}(\alpha)-\psi_{i o}^{-1}(1-\alpha) \leq 0 \quad i=1, \cdots, m,
\end{aligned}
$$

$$
\begin{array}{ll}
y_{r}-\sum_{j=1}^{n} \chi_{r j}^{-1}(1-\alpha) \lambda_{j} \leq 0 & r=1, \cdots, s, \\
\lambda_{j} \geq 0, \quad y_{r} \geq 0 \quad j=1, \cdots, n, & r=1, \cdots, s .
\end{array}
$$

Using the weighting method, the uncertain multiobjective (Model (35)) is rewritten as follows:

$$
\begin{aligned}
& \min \left(-w_{1} Z_{1}-w_{2} Z_{2}\right) \\
& \text { s.t. } \\
& \sum_{j=1}^{n} \lambda_{j} \psi_{i j}^{-1}(\alpha)-\psi_{i o}^{-1}(1-\alpha) \leq 0 \quad i=1, \cdots, m, \\
& y_{r}-\sum_{j=1}^{n} \chi_{r j}^{-1}(1-\alpha) \lambda_{j} \leq 0 \\
& \lambda_{j} \geq 0, \quad y_{r} \geq 0 \quad j=1, \cdots, n, \quad r=1, \cdots, s .
\end{aligned}
$$

Definition 19. The greater the optimal objective value in Model (36) is, the more efficient is the $D M U_{o}$.

Using the uncertain entropy model as the second objective function, we will be able to achieve an optimal performance in cost and revenue models with the maximum dispersion rate in their constituent components. As a solution methodology, the uncertain allocation models are converted to crisp models using the two EV approaches. The uncertain multi-objective cost efficiency model is introduced using the EV method as follows. The EV method is used for converting the new model to a crisp model.

$$
\begin{aligned}
& Z_{1}=\min \sum_{i=1}^{m} x_{i} \int_{0}^{1} \Phi_{i o}^{-1}(\alpha) d \alpha \\
& Z_{2}=\max \sum_{i=1}^{m} x_{i} \int_{0}^{1} \Phi_{i o}^{-1}(\alpha) \ln \frac{\alpha}{1-\alpha} d \alpha
\end{aligned}
$$

s.t.:

$$
\begin{aligned}
& \sum_{j=1}^{n} \int_{0}^{1} \psi_{i j}^{-1}(\alpha) \lambda_{j} d \alpha-x_{i} \leq 0 \quad i=1, \cdots, m, \\
& \int_{0}^{1} \chi_{r o}^{-1}(\alpha) d \alpha-\sum_{j=1}^{n} \int_{0}^{1} \chi_{r j}^{-1}(1-\alpha) \lambda_{j} d \alpha \leq 0 \\
& r=1, \cdots, s, \\
& \lambda_{j} \geq 0, \quad x_{i} \geq 0 \quad j=1, \cdots, n, \quad i=1, \cdots, m .
\end{aligned}
$$


Using the weighting method, the uncertain multiobjective (Model (37)) is rewritten as follows:

$$
\begin{aligned}
& \min \left(w_{1} Z_{1}-w_{2} Z_{2}\right), \\
& \text { s.t.: } \\
& \sum_{j=1}^{n} \int_{0}^{1} \psi_{i j}^{-1}(\alpha) \lambda_{j} d \alpha-x_{i} \leq 0 \quad i=1, \cdots, m, \\
& \int_{0}^{1} \chi_{r o}^{-1}(\alpha) d \alpha-\sum_{j=1}^{n} \int_{0}^{1} \chi_{r j}^{-1}(1-\alpha) \lambda_{j} d \alpha \leq 0 \\
& \quad r=1, \cdots, s,
\end{aligned}
$$$$
\lambda_{j} \geq 0, \quad x_{i} \geq 0 \quad j=1, \cdots, n, \quad i=1, \cdots, m .
$$

Definition 20 (efficiency). $D M U_{o}$ is efficient if and only if the optimal solution in Model (38) can achieve the value of 1 .

Using the EV method, the multi-objective revenue efficiency model consisting of an uncertain revenue model and an uncertain entropy is introduced as follows:

$$
\begin{aligned}
& Z_{1}=\max \sum_{r=1}^{s} y_{r} \int_{0}^{1} \Psi_{r o}^{-1}(\alpha) d \alpha \\
& Z_{2}=\max \sum_{r=1}^{s} y_{r} \int_{0}^{1} \Psi_{r o}^{-1}(\alpha) \ln \left(\frac{\alpha}{1-\alpha}\right) d \alpha
\end{aligned}
$$

s.t.:

$$
\begin{aligned}
& \sum_{j=1}^{n} \lambda_{j} \int_{0}^{1} \psi_{i j}^{-1}(\alpha) d \alpha-\int_{0}^{1} \psi_{i o}^{-1}(1-\alpha) d \alpha \leq 0 \\
& i=1, \cdots, m, \\
& y_{r}-\sum_{j=1}^{n} \lambda_{j} \int_{0}^{1} \chi_{r j}^{-1}(1-\alpha) \leq 0 \quad r=1, \cdots, s, \\
& \lambda_{j} \geq 0, \quad y_{r} \geq 0 \quad j=1, \cdots, n, \quad r=1, \cdots, s .
\end{aligned}
$$

Using the weighting method, the uncertain multiobjective (Model (37)) is rewritten as follows:

$$
\min \left(-w_{1} Z_{1}-w_{2} Z_{2}\right)
$$

s.t.:

$$
\begin{aligned}
& \sum_{j=1}^{n} \lambda_{j} \int_{0}^{1} \psi_{i j}^{-1}(\alpha) d \alpha-\int_{0}^{1} \psi_{i o}^{-1}(1-\alpha) d \alpha \leq 0 \\
& i=1, \cdots, m, \\
& y_{r}-\sum_{j=1}^{n} \lambda_{j} \int_{0}^{1} \chi_{r j}^{-1}(1-\alpha) \leq 0 \quad r=1, \cdots, s, \\
& \lambda_{j} \geq 0, \quad y_{r} \geq 0 \quad j=1, \cdots, n, \quad r=1, \cdots, s .
\end{aligned}
$$

Definition 21 (efficiency). $D M U_{o}$ will be efficient if and only if the optimal solution in Model (40) can achieve the value of 1 .

\section{Practical example}

The accuracy of the above-mentioned models is examined using a practical example. There is a wellknown direct relationship between business in stock market and market forecasting. In the meantime, the use of beliefs and opinions of experts in the field of buying and selling stocks is of great importance to obtaining the maximum revenue at the minimum cost. Therefore, the use of belief theory in the stock market to take into account the most efficient suggestions will be helpful in this business. Tables 1 and 2 list data of 25 stockbrokers for buying and selling a same stock portfolio with different prices. Considering that the amounts and numbers of stock are equal and the buying and selling prices are uncertain variables (Tables 3 and 4), the efficiency of the cost and revenue models in the stock portfolio is explained.

The results obtained from Model (34) with different $w_{1}$ and $w_{2}$ are presented in Table 5 .

According to the results, none of the cost-efficient DMUs is equal to 1. According to Definition (18), a larger optimal objective value means a more efficient $D M U_{o}$. Therefore, according to Model (34):

1. If $w_{1}=0.6, w_{2}=0.4$, then $D M U_{17}$ is efficient;

2. If $w_{1}=0.7, w_{2}=0.3$, then $D M U_{8}$ is efficient;

3. If $w_{1}=0.8, w_{2}=0.2$, then $D M U_{8}$ is efficient;

4. If $w_{1}=0.9, w_{2}=0.1$, then $D M U_{8}$ is efficient.

The results obtained from Model (38) with different $w_{1}$ and $w_{2}$ are shown in Table 6.

As clearly seen, the cost-efficient DMUs are equal to 1 . According to Definition 20 and Model (38):

1. If $w_{1}=0.6, w_{2}=0.4$, then $D M U_{17}$ is efficient;

2. If $w_{1}=0.7, w_{2}=0.3$, then $D M U_{8}$ is efficient;

3. If $w_{1}=0.8, w_{2}=0.2$, then $D M U_{4}, D M U_{7}, D M U_{8}$, $D M U_{11}, D M U_{16}$, and $D M U_{17}$ are efficient. 
Table 1. DMUs with three uncertain inputs $\left(x_{i j}\right)$.

\begin{tabular}{|c|c|c|c|}
\hline$D M U_{i}$ & Iran-Khodro & Saderat Bank & Pars Petrochemical \\
\hline$D M U_{1}$ & $Z(23200,24300,26400)$ & $Z(26300,27400,28600)$ & $Z(19000,19900,20200)$ \\
\hline$D M U_{2}$ & $Z(20000,22000,240000)$ & $Z(27600,28900,29000)$ & $Z(18500,19300,21200)$ \\
\hline$D M U_{3}$ & $Z(21000,22500,24300)$ & $Z(28300,29500,31200)$ & $Z(22000,23200,24100)$ \\
\hline $\mathrm{DMU}_{4}$ & $Z(21500,22700,23300)$ & $Z(31200,32700,34200)$ & $Z(17100,17900,18600)$ \\
\hline$D M U_{5}$ & $Z(22900,25000,26300)$ & $Z(33100,34300,36100)$ & $Z(22300,23300,24000)$ \\
\hline$D M U_{6}$ & $Z(21200,23200,24500)$ & $Z(25230,25740,26150)$ & $Z(18340,18770,18960)$ \\
\hline$D M U_{7}$ & $Z(22300,23300,24900)$ & $Z(26150,26630,26970)$ & $Z(17000,17300,17700)$ \\
\hline$D M U_{8}$ & $Z(21700,22900,23700)$ & $Z(27200,27730,28050)$ & $Z(18100,18900,19150)$ \\
\hline$D M U_{9}$ & $Z(23100,23800,24100)$ & $Z(27330,27520,27740)$ & $Z(19200,19740,20000)$ \\
\hline$D M U_{10}$ & $Z(21100,21700,22300)$ & $Z(28130,28430,28720)$ & $Z(22350,22980,23110)$ \\
\hline$D M U_{11}$ & $Z(22400,23200,24300)$ & $Z(29220,29780,30050)$ & $Z(18100,18820,19100)$ \\
\hline$D M U_{12}$ & $Z(23200,23900,24500)$ & $Z(26130,26740,26970)$ & $Z(19230,19490,19990)$ \\
\hline$D M U_{13}$ & $Z(22200,22700,23200)$ & $Z(26000,26370,26700)$ & $Z(21340,21780,22000)$ \\
\hline$D M U_{14}$ & $Z(23100,23500,23900)$ & $Z(28240,28530,28780)$ & $Z(22270,22520,22990)$ \\
\hline$D M U_{15}$ & $Z(22200,22900,23200)$ & $Z(25430,25790,26100)$ & $Z(23410,23800,24000)$ \\
\hline$D M U_{16}$ & $Z(21400,21700,21800)$ & $Z(27200,27730,28050)$ & $Z(19180,19900,22000)$ \\
\hline$D M U_{17}$ & $Z(20100,20700,21200)$ & $Z(26050,26390,26430)$ & $Z(18170,18890,19220)$ \\
\hline$D M U_{18}$ & $Z(20300,20800,21300)$ & $Z(27300,27800,27990)$ & $Z(21310,21660,21960)$ \\
\hline$D M U_{19}$ & $Z(21000,21300,21800)$ & $Z(25130,25640,25830)$ & $Z(19730,20000,20150)$ \\
\hline$D M U_{20}$ & $Z(22300,22900,23200)$ & $Z(25000,25390,25930)$ & $Z(22110,22730,23100)$ \\
\hline$D M U_{21}$ & $Z(20150,20400,21000)$ & $Z(26170,26600,26820)$ & $Z(23430,23730,24150)$ \\
\hline$D M U_{22}$ & $Z(21100,21650,22100)$ & $Z(27230,27470,27580)$ & $Z(21210,21840,22050)$ \\
\hline$D M U_{23}$ & $Z(22300,22750,22960)$ & $Z(31100,31430,31690)$ & $Z(22120,22590,22970)$ \\
\hline$D M U_{24}$ & $Z(22150,22230,22780)$ & $Z(32330,32560,32810)$ & $Z(23380,23980,24400)$ \\
\hline
\end{tabular}

Table 7 presents the results obtained from Model (36) with different $w_{1}$ and $w_{2}$.

According to the results, none of the revenueefficient DMUs is equal to 1 . According to Definition 19, a larger optimal objective value means a more efficient $D M U_{o}$. Therefore, according to Model (36):
1. If $w_{1}=0.6, w_{2}=0.4$, then $D M U_{6}, D M U_{7}, D M U_{8}$, $D M U_{17}, D M U_{19}, D M U_{20}$, and $D M U_{21}$ are efficient;

2. If $w_{1}=0.5, w_{2}=0.5$, then $D M U_{6}, D M U_{7}, D M U_{8}$, $D M U_{17}, D M U_{19}, D M U_{20}$, and $D M U_{21}$ are efficient.

The results obtained from Model (40) with different $w_{1}$ and $w_{2}$ are presented in Table 8. 
Table 2. DMUs with three uncertain outputs $\left(y_{i j}\right)$.

\begin{tabular}{|c|c|c|c|}
\hline$D M U_{i}$ & Iran-Khodro & Saderat Bank & Pars Petrochemical \\
\hline$D M U_{1}$ & $Z(17500,18100,19600)$ & $Z(23500,25000,26100)$ & $Z(12000,12500,13200)$ \\
\hline$D M U_{2}$ & $Z(15100,16300,20000)$ & $Z(25000,26100,27300)$ & $Z(13400,17800,18400)$ \\
\hline$D M U_{3}$ & $Z(19000,20000,20900)$ & $Z(24200,25700,27600)$ & $Z(17300,18400,20000)$ \\
\hline$D M U_{4}$ & $Z(16000,17300,20000)$ & $Z(28100,29300,30300)$ & $Z(13000,13400,14100)$ \\
\hline$D M U_{5}$ & $Z(18400,19200,24000)$ & $Z(24000,25100,26500)$ & $Z(17300,18500,20500)$ \\
\hline$D M U_{6}$ & $Z(16200,16900,17200)$ & $Z(26230,26470,26580)$ & $Z(14110,14730,15100)$ \\
\hline$D M U_{7}$ & $Z(19100,19700,20200)$ & $Z(23130,23640,23830)$ & $Z(12410,12800,13000)$ \\
\hline$D M U_{8}$ & $Z(17100,17700,18300)$ & $Z(26430,26790,27100)$ & $Z(17230,17490,17990)$ \\
\hline$D M U_{9}$ & $Z(15100,15650,16100)$ & $Z(28130,28740,28970)$ & $Z(13210,13840,14050)$ \\
\hline$D M U_{10}$ & $Z(18050,18250,18530)$ & $Z(23330,23520,23740)$ & $Z(15310,15660,15960)$ \\
\hline$D M U_{11}$ & $Z(17300,17750,17960)$ & $Z(28240,28530,28780)$ & $Z(14340,14780,15000)$ \\
\hline$D M U_{12}$ & $Z(16000,16300,16800)$ & $Z(24300,24800,24990)$ & $Z(12230,12540,12870)$ \\
\hline$D M U_{13}$ & $Z(19150,19400,20000)$ & $Z(25170,25660,25820)$ & $Z(13180,13900,14000)$ \\
\hline$D M U_{14}$ & $Z(15200,15900,16500)$ & $Z(24230,24740,25150)$ & $Z(15700,15900,16150)$ \\
\hline$D M U_{15}$ & $Z(18300,19300,20900)$ & $Z(24200,24730,25050)$ & $Z(12730,13000,13150)$ \\
\hline$D M U_{16}$ & $Z(19400,20200,21300)$ & $Z(23000,23370,23700)$ & $Z(16380,16980,17400)$ \\
\hline$D M U_{17}$ & $Z(16100,16500,16900)$ & $Z(22200,22730,23050)$ & $Z(17340,17770,18960)$ \\
\hline$D M U_{18}$ & $Z(18300,18900,19200)$ & $Z(23000,23390,23930)$ & $Z(13170,13890,14220)$ \\
\hline$D M U_{19}$ & $Z(17150,17230,17780)$ & $Z(28330,28560,28810)$ & $Z(14430,14730,15150)$ \\
\hline$D M U_{20}$ & $Z(16300,16800,17300)$ & $Z(24220,24780,25050)$ & $Z(12350,12980,13110)$ \\
\hline$D M U_{21}$ & $Z(19400,19700,19800)$ & $Z(22100,22430,22690)$ & $Z(13100,13820,14100)$ \\
\hline$D M U_{22}$ & $Z(19200,19700,20200)$ & $Z(25130,25430,25720)$ & $Z(16120,16590,16970)$ \\
\hline$D M U_{23}$ & $Z(16100,16800,17100)$ & $Z(28030,28340,28620)$ & $Z(17270,17520,17990)$ \\
\hline$D M U_{24}$ & $Z(18700,19900,20700)$ & $Z(25050,25390,25430)$ & $Z(14000,14300,14770)$ \\
\hline$D M U_{25}$ & $Z(15200,16200,17500)$ & $Z(23150,23630,23970)$ & $Z(15600,15980,16430)$ \\
\hline
\end{tabular}

Obviously, the cost-efficient DMUs are equal to 1. According to Definition 21 and Model (40):

1. If $w_{1}=0.3, w_{2}=0.7$, then $D M U_{3}, D M U_{8}, D M U_{16}$, and $D M U_{17}$ are efficient;

2. If $w_{1}=0.4, w_{2}=0.6$, then $D M U_{8}, D M U_{17}$,
$D M U_{18}, \quad D M U_{19}, \quad D M U_{20}, \quad D M U_{21}, \quad D M U_{22}$, $D M U_{23}, D M U_{24}$, and $D M U_{25}$ are efficient;

3. If $w_{1}=0.5, w_{2}=0.5$, then $D M U_{2}, D M U_{3}, D M U_{6}$, $D M U_{8}, D M U_{11}, D M U_{16}, D M U_{17}, D M U_{19}, D M U_{22}$, and $D M U_{23}$ are efficient. 
Table 3. Input costs for DMUs $\left(c_{i j}\right)$.

\begin{tabular}{|c|c|c|c|}
\hline$D M U_{i}$ & Iran-Khodro & Saderat Bank & Pars Petrochemical \\
\hline$D M U_{1}$ & $Z(2603,2610,2715)$ & $Z(950,1000,1100)$ & $Z(30000,32000,34000)$ \\
\hline$D M U_{2}$ & $Z(2500,2520,2580)$ & $Z(900,950,990)$ & $Z(28000,29000,30000)$ \\
\hline$D M U_{3}$ & $Z(2450,2500,2530)$ & $Z(800,900,910)$ & $Z(27000,27500,27900)$ \\
\hline$D M U_{4}$ & $Z(2300,2310,2340)$ & $Z(810,820,850)$ & $Z(26000,26500,26700)$ \\
\hline$D M U_{5}$ & $Z(2200,2250,2290)$ & $Z(840,850,869)$ & $Z(25000,25500,25800)$ \\
\hline$D M U_{6}$ & $Z(2000,2100,2200)$ & $Z(890,899,9100)$ & $Z(24000,24900,25100)$ \\
\hline$D M U_{7}$ & $Z(2190,2220,2240)$ & $Z(800,810,830)$ & $Z(28500,28600,29100)$ \\
\hline$D M U_{8}$ & $Z(2199,2210,2299)$ & $Z(830,840,850)$ & $Z(29100,29250,29380)$ \\
\hline$D M U_{9}$ & $Z(2090,2099,2115)$ & $Z(840,850,860)$ & $Z(29500,29900,30000)$ \\
\hline$D M U_{10}$ & $Z(2100,2150,2180)$ & $Z(830,890,920)$ & $Z(30000,31000,31990)$ \\
\hline$D M U_{11}$ & $Z(2110,2120,2130)$ & $Z(910,960,990)$ & $Z(31000,32000,32850)$ \\
\hline$D M U_{12}$ & $Z(2122,2139,2169)$ & $Z(990,1010,1030)$ & $Z(32000,32800,33500)$ \\
\hline$D M U_{13}$ & $Z(2220,2270,2299)$ & $Z(1000,1019,1038)$ & $Z(33000,33790,33990)$ \\
\hline$D M U_{14}$ & $Z(2000,2050,2091)$ & $Z(1100,1200,1290)$ & $Z(33000,33900,34100)$ \\
\hline$D M U_{15}$ & $Z(1900,1960,1999)$ & $Z(1090,1099,1120)$ & $Z(34000,34900,35200)$ \\
\hline$D M U_{16}$ & $Z(2070,2080,2090)$ & $Z(1190,1200,1210)$ & $Z(35000,35990,36590)$ \\
\hline$D M U_{17}$ & $Z(2120,2160,2190)$ & $Z(1210,1219,1229)$ & $Z(35900,36200,36990)$ \\
\hline$D M U_{18}$ & $Z(2198,2210,2250)$ & $Z(1290,1231,1391)$ & $Z(36000,36900,37000)$ \\
\hline$D M U_{19}$ & $Z(2260,2290,2300)$ & $Z(1391,1399,1410)$ & $Z(37000,37500,37990)$ \\
\hline$D M U_{20}$ & $Z(2310,2321,2347)$ & $Z(1290,1310,1380)$ & $Z(37700,38100,38800)$ \\
\hline$D M U_{21}$ & $Z(2340,2370,2398)$ & $Z(1170,1199,1270)$ & $Z(38000,38200,38400)$ \\
\hline$D M U_{22}$ & $Z(2410,2419,2421)$ & $Z(1280,1320,1410)$ & $Z(38600,38900,38990)$ \\
\hline$D M U_{23}$ & $Z(2380,2391,2400)$ & $Z(1470,1499,1510)$ & $Z(38710,38790,38990)$ \\
\hline$D M U_{24}$ & $Z(2330,2340,2350)$ & $Z(1510,1590,1690)$ & $Z(40000,41000,42500)$ \\
\hline$D M U_{25}$ & $Z(2410,2427,2439)$ & $Z(1700,1820,1899)$ & $Z(43000,45000,45990)$ \\
\hline
\end{tabular}

It seems that $D M U_{8}$ and $D M U_{17}$ have the best performance for selling a same stock portfolio with different prices.

According to the above results, $D M U_{8}$ and $D M U_{17}$ seem to be efficient in most models. These two DMUs showed the best performance in both cost and revenue models for buying and selling the same stock portfolio with different prices.

\section{Conclusion}

This paper was aimed at presenting an uncertain allocation model with inherent complexity of uncertain models. Due to the complexity of the new models, two methods were proposed to convert the uncertain models into crisp models. Finally, an real example from the Iranian stock market was used to examine 
Table 4. Output prices for DMUs $\left(p_{i j}\right)$.

\begin{tabular}{|c|c|c|c|}
\hline$D M U_{i}$ & Iran-Khodro & Saderat Bank & Pars Petrochemical \\
\hline$D M U_{1}$ & $Z(17500,18100,19600)$ & $Z(23500,25000,26100)$ & $Z(12000,12500,13200)$ \\
\hline$D M U_{2}$ & $Z(15100,16300,20000)$ & $Z(25000,26100,27300)$ & $Z(13400,17800,18400)$ \\
\hline$D M U_{3}$ & $Z(19000,20000,20900)$ & $Z(24200,25700,27600)$ & $Z(17300,18400,20000)$ \\
\hline$D M U_{4}$ & $Z(16000,17300,20000)$ & $Z(28100,29300,30300)$ & $Z(13000,13400,14100)$ \\
\hline$D M U_{5}$ & $Z(18400,19200,24000)$ & $Z(24000,25100,26500)$ & $Z(17300,18500,20500)$ \\
\hline$D M U_{6}$ & $Z(16200,16900,17200)$ & $Z(26230,26470,26580)$ & $Z(14110,14730,15100)$ \\
\hline$D M U_{7}$ & $Z(19100,19700,20200)$ & $Z(23130,23640,23830)$ & $Z(12410,12800,13000)$ \\
\hline$D M U_{8}$ & $Z(17100,17700,18300)$ & $Z(26430,26790,27100)$ & $Z(17230,17490,17990)$ \\
\hline$D M U_{9}$ & $Z(15100,15650,16100)$ & $Z(28130,28740,28970)$ & $Z(13210,13840,14050)$ \\
\hline$D M U_{10}$ & $Z(18050,18250,18530)$ & $Z(23330,23520,23740)$ & $Z(15310,15660,15960)$ \\
\hline$D M U_{11}$ & $Z(17300,17750,17960)$ & $Z(28240,28530,28780)$ & $4780,15000)$ \\
\hline$D M U_{12}$ & $Z(16000,16300,16800)$ & $Z(24300,24800,24990)$ & $Z(12230,12540,12870)$ \\
\hline$D M U_{13}$ & $Z(19150,19400,20000)$ & $Z(25170,25660,25820)$ & $Z(13180,13900,14000)$ \\
\hline$D M U_{14}$ & $Z(15200,15900,16500)$ & $Z(24230,24740,25150)$ & $Z(15700,15900,16150)$ \\
\hline$D M U_{15}$ & $Z(18300,19300,20900)$ & $Z(24200,24730,25050)$ & $Z(12730,13000,13150)$ \\
\hline$D M U_{16}$ & $Z(19400,20200,21300)$ & $Z(23000,23370,23700)$ & $Z(16380,16980,17400)$ \\
\hline$D M U_{17}$ & $Z(16100,16500,16900)$ & $Z(22200,22730,23050)$ & $Z(17340,17770,18960)$ \\
\hline$D M U_{18}$ & $Z(18300,18900,19200)$ & $Z(23000,23390,23930)$ & $Z(13170,13890,14220)$ \\
\hline$D M U_{19}$ & $Z(17150,17230,17780)$ & $Z(28330,28560,28810)$ & $Z(14430,14730,15150)$ \\
\hline$D M U_{20}$ & $Z(16300,16800,17300)$ & $Z(24220,24780,25050)$ & $Z(12350,12980,13110)$ \\
\hline$D M U_{21}$ & $Z(19400,19700,19800)$ & $Z(22100,22430,22690)$ & $Z(13100,13820,14100)$ \\
\hline$D M U_{22}$ & $Z(19200,19700,20200)$ & $Z(25130,25430,25720)$ & $Z(16120,16590,16970)$ \\
\hline$D M U_{23}$ & $Z(16100,16800,17100)$ & $Z(28030,28340,28620)$ & $Z(17270,17520,17990)$ \\
\hline$D M U_{24}$ & $Z(18700,19900,20700)$ & $Z(25050,25390,25430)$ & $Z(14000,14300,14770)$ \\
\hline$D M U_{25}$ & $Z(15200,16200,17500)$ & $Z(23150,23630,23970)$ & $Z(15600,15980,16430)$ \\
\hline
\end{tabular}

the performance of the new models. For this purpose, 25 stockbrokers were selected to determine buying and selling prices of a single stock portfolio with different prices in the cost and revenue models. The amounts and numbers of stock were considered to be equal.
The buying and selling prices were also considered as uncertain variables. The presented models can help managers choose the best portfolio in the stock market. Instead of the expected values for objective functions with uncertain variables, the variance can be taken into 
Table 5. The results of evaluating cost efficiency with Model (34) $(\alpha=0.5)$.

\begin{tabular}{|c|c|c|c|c|c|}
\hline$D M U_{i}$ & $D M U_{1}$ & $D M U_{2}$ & $D M U_{3}$ & $D_{M} U_{4}$ & $D M U_{5}$ \\
\hline$w_{1}=0.6, w_{2}=0.4$ & 0.149 & 0.168 & 0.156 & 0.170 & 0.150 \\
\hline$w_{1}=0.7, w_{2}=0.3$ & 0.324 & 0.370 & 0.342 & 0.375 & 0.330 \\
\hline$w_{1}=0.8, w_{2}=0.2$ & 0.504 & 0.571 & 0.527 & 0.581 & 0.513 \\
\hline$w_{1}=0.9, w_{2}=0.1$ & 0.687 & 0.770 & 0.718 & 0.790 & 0.694 \\
\hline$D M U_{i}$ & $D M U_{6}$ & $D M U_{7}$ & $D M U_{8}$ & $D M U_{9}$ & $D M U_{10}$ \\
\hline$w_{1}=0.6, w_{2}=0.4$ & 0.164 & 0.170 & 0.172 & 0.158 & 0.139 \\
\hline$w_{1}=0.7, w_{2}=0.3$ & 0.361 & 0.376 & 0380 & 0.347 & 0.303 \\
\hline$w_{1}=0.8, w_{2}=0.2$ & 0.556 & 0.558 & 0.585 & 0.539 & 0.471 \\
\hline$w_{1}=0.9, w_{2}=0.1$ & 0.753 & 0.790 & 0.799 & 0.728 & 0.640 \\
\hline$D M U_{i}$ & $D M U_{11}$ & $D M U_{12}$ & $D M U_{13}$ & $D M U_{14}$ & $D M U_{15}$ \\
\hline$w_{1}=0.6, w_{2}=0.4$ & 0.168 & 0.144 & 0.146 & 0.133 & 0.130 \\
\hline$w_{1}=0.7, w_{2}=0.3$ & 0.370 & 0.314 & 0.320 & 0.293 & 0.285 \\
\hline$w_{1}=0.8, w_{2}=0.2$ & 0.575 & 0.488 & 0.496 & 0.455 & 0.442 \\
\hline$w_{1}=0.9, w_{2}=0.1$ & 0.777 & 0.664 & 0.675 & 0.618 & 0.601 \\
\hline$D M U_{i}$ & $D M U_{16}$ & $D M U_{17}$ & $D M U_{18}$ & $D M U_{19}$ & $D M U_{20}$ \\
\hline$w_{1}=0.6, w_{2}=0.4$ & 0.168 & 0.173 & 0.142 & 0.160 & 0.128 \\
\hline$w_{1}=0.7, w_{2}=0.3$ & 0.370 & 0.377 & 0.310 & 0.352 & 0.282 \\
\hline$w_{1}=0.8, w_{2}=0.2$ & 0.574 & 0.584 & 0.481 & .0 .545 & 0.438 \\
\hline$w_{1}=0.9, w_{2}=0.1$ & 0.781 & 0.795 & 0.654 & 0.742 & 0.595 \\
\hline$D M U_{i}$ & $D M U_{21}$ & $D M U_{22}$ & $D M U_{23}$ & $D M U_{24}$ & $D M U_{25}$ \\
\hline$w_{1}=0.6, w_{2}=0.4$ & 0.133 & 0.155 & 0.150 & 0.135 & 0.132 \\
\hline$w_{1}=0.7, w_{2}=0.3$ & 0.291 & 0.340 & 0.329 & 0.294 & 0.289 \\
\hline$w_{1}=0.8, w_{2}=0.2$ & 0.451 & 0.528 & 0.510 & 0.456 & 0.450 \\
\hline$w_{1}=0.9, w_{2}=0.1$ & 0.617 & 0.718 & 0.693 & 0.619 & 0.611 \\
\hline
\end{tabular}

Table 6. The result of evaluating cost efficiency with Model (38) $(\alpha=0.5)$.

\begin{tabular}{|c|c|c|c|c|c|}
\hline$D M U_{i}$ & $D M U_{1}$ & $D M U_{2}$ & $D M U_{3}$ & $\mathrm{DMU}_{4}$ & $D M U_{5}$ \\
\hline$w_{1}=0.6, w_{2}=0.4$ & 0.262 & 0.287 & 0.274 & 0.300 & 0.267 \\
\hline$w_{1}=0.7, w_{2}=0.3$ & 0.570 & 0.628 & 0.600 & 0.656 & 0.587 \\
\hline$w_{1}=0.8, w_{2}=0.2$ & 0.882 & 0.965 & 0.921 & 1 & 0.907 \\
\hline$D M U_{i}$ & $D M U_{6}$ & $D M U_{7}$ & $D M U_{8}$ & $D M U_{9}$ & $D M U_{10}$ \\
\hline$w_{1}=0.6, w_{2}=0.4$ & 0.285 & 0.297 & 0.301 & 0.275 & 0.244 \\
\hline$w_{1}=0.7, w_{2}=0.3$ & 0.627 & 0.651 & 0.663 & 0.605 & 0.532 \\
\hline$w_{1}=0.8, w_{2}=0.2$ & 0.966 & 1 & 1 & 0.939 & 0.826 \\
\hline$D M U_{i}$ & $D M U_{11}$ & $D M U_{12}$ & $D M U_{13}$ & $D M U_{14}$ & $D M U_{15}$ \\
\hline$w_{1}=0.6, w_{2}=0.4$ & 0.294 & 0.252 & 0.255 & 0.233 & 0.228 \\
\hline$w_{1}=0.7, w_{2}=0.3$ & 0.645 & 0.548 & 0.559 & 0.511 & 0.500 \\
\hline$w_{1}=0.8, w_{2}=0.2$ & 1 & 0.850 & 0.867 & 0.791 & 0.773 \\
\hline$D M U_{i}$ & $D M U_{16}$ & $D M U_{17}$ & $D M U_{18}$ & $D M U_{19}$ & $D M U_{20}$ \\
\hline$w_{1}=0.6, w_{2}=0.4$ & 0.298 & 0.302 & 0.248 & 0.280 & 0.225 \\
\hline$w_{1}=0.7, w_{2}=0.3$ & 0.653 & 0.657 & 0.543 & 0.614 & 0.492 \\
\hline$w_{1}=0.8, w_{2}=0.2$ & 1 & 1 & 0.838 & 0.952 & 0.761 \\
\hline$D M U_{i}$ & $D M U_{21}$ & $D M U_{22}$ & $D M U_{23}$ & $D M U_{24}$ & $D M U_{25}$ \\
\hline$w_{1}=0.6, w_{2}=0.4$ & 0.233 & 0.273 & 0.262 & 0.236 & 0.231 \\
\hline$w_{1}=0.7, w_{2}=0.3$ & 0.510 & 0.598 & 0.574 & 0.515 & 0.502 \\
\hline$w_{1}=0.8, w_{2}=0.2$ & 0.788 & 0.927 & 0.890 & 0.796 & 0.783 \\
\hline
\end{tabular}


Table 7. The result of evaluating revenue efficiency with Model (36) $(\alpha=0.5)$.

\begin{tabular}{|c|c|c|c|c|c|}
\hline$D M U_{i}$ & $D M U_{1}$ & $D M U_{2}$ & $D_{M} U_{3}$ & $\mathrm{DMU}_{4}$ & $D M U_{5}$ \\
\hline$w_{1}=0.4, w_{2}=0.6$ & 0.51 & 0.59 & 0.63 & 0.59 & 0.57 \\
\hline$w_{1}=0.5, w_{2}=0.5$ & 0.51 & 0.73 & 0.78 & 0.76 & 0.73 \\
\hline$D M U_{i}$ & $D M U_{6}$ & $D M U_{7}$ & $D M U_{8}$ & $D M U_{9}$ & $D M U_{10}$ \\
\hline$w_{1}=0.4, w_{2}=0.6$ & 0.71 & 0.71 & 0.71 & 0.53 & 0.58 \\
\hline$w_{1}=0.5, w_{2}=0.5$ & 0.82 & 0.82 & 0.82 & 0.67 & 0.73 \\
\hline$D M U_{i}$ & $D M U_{11}$ & $D M U_{12}$ & $D M U_{13}$ & $D M U_{14}$ & $D M U_{15}$ \\
\hline$w_{1}=0.4, w_{2}=0.6$ & 0.57 & 0.50 & 0.54 & 0.55 & 0.55 \\
\hline$w_{1}=0.5, w_{2}=0.5$ & 0.74 & 0.62 & 0.69 & 0.69 & 0.71 \\
\hline$D M U_{i}$ & $D M U_{16}$ & $D M U_{17}$ & $D M U_{18}$ & $D M U_{19}$ & $D M U_{20}$ \\
\hline$w_{1}=0.4, w_{2}=0.6$ & 0.62 & 0.71 & 0.53 & 0.71 & 0.71 \\
\hline$w_{1}=0.5, w_{2}=0.5$ & 0.73 & 0.82 & 0.68 & 0.82 & 0.82 \\
\hline$D M U_{i}$ & $D M U_{21}$ & $D M U_{22}$ & $D M U_{23}$ & $D M U_{24}$ & $D M U_{25}$ \\
\hline$w_{1}=0.4, w_{2}=0.6$ & 0.71 & 0.60 & 0.59 & 0.51 & 0.53 \\
\hline$w_{1}=0.5, w_{2}=0.5$ & 0.82 & 0.73 & 0.73 & 0.67 & 0.67 \\
\hline
\end{tabular}

Table 8. The result of evaluating revenue efficiency with Model (40) ( $\alpha=0.5)$.

\begin{tabular}{|c|c|c|c|c|c|}
\hline$D M U_{i}$ & $D M U_{1}$ & $D M U_{2}$ & $D M U_{3}$ & $D M U_{4}$ & $D M U_{5}$ \\
\hline$w_{1}=0.3, w_{2}=0.7$ & 0.81 & 0.95 & 1 & 0.90 & 0.93 \\
\hline$w_{1}=0.4, w_{2}=0.6$ & 0.83 & 0.93 & 0.81 & 0.915 & 0.87 \\
\hline$w_{1}=0.5, w_{2}=0.5$ & 0.92 & 1 & 1 & 0.955 & 0.977 \\
\hline$D M U_{i}$ & $D M U_{6}$ & $D M U_{7}$ & $D M U_{8}$ & $D M U_{9}$ & $D M U_{10}$ \\
\hline$w_{1}=0.3, w_{2}=0.7$ & 0.98 & 0.92 & 1 & 0.83 & 0.93 \\
\hline$w_{1}=0.4, w_{2}=0.6$ & 0.915 & 0.885 & 0.97 & 0.87 & 0.905 \\
\hline$w_{1}=0.5, w_{2}=0.5$ & 1 & 0.963 & 1 & 0.946 & 0.963 \\
\hline$D M U_{i}$ & $D M U_{11}$ & $D M U_{12}$ & $D M U_{13}$ & $D M U_{14}$ & $D M U_{15}$ \\
\hline$w_{1}=0.3, w_{2}=0.7$ & 0.98 & 0.89 & 0.90 & 0.92 & 0.88 \\
\hline$w_{1}=0.4, w_{2}=0.6$ & 0.905 & 0.85 & 0.89 & 0.89 & 0.86 \\
\hline$w_{1}=0.5, w_{2}=0.5$ & 1 & 0.937 & 0.963 & 0.955 & 0.946 \\
\hline$D M U_{i}$ & $D M U_{16}$ & $D M U_{17}$ & $D M U_{18}$ & $D M U_{19}$ & $D M U_{20}$ \\
\hline$w_{1}=0.3, w_{2}=0.7$ & 1 & 1 & 0.90 & 0.98 & 0.92 \\
\hline$w_{1}=0.4, w_{2}=0.6$ & 0.93 & 1 & 1 & 1 & 1 \\
\hline$w_{1}=0.5, w_{2}=0.5$ & 1 & 1 & 0.955 & 1 & 0.955 \\
\hline$D M U_{i}$ & $D M U_{21}$ & $D M U_{22}$ & $D M U_{23}$ & $D M U_{24}$ & $D M U_{25}$ \\
\hline$w_{1}=0.3, w_{2}=0.7$ & 0.93 & 0.94 & 0.89 & 0.83 & 0.83 \\
\hline$w_{1}=0.4, w_{2}=0.6$ & 1 & 1 & 1 & 1 & 1 \\
\hline$w_{1}=0.5, w_{2}=0.5$ & 0.963 & 1 & 1 & 0.955 & 0.946 \\
\hline
\end{tabular}

account in future studies. The problem presented in this paper can be also studied with normal uncertain variables.

\section{References}

1. Charnes, A., Cooper, W.W., and Rhodes, E. "Measuring the efficiency of decision making units", European Journal of Operational Research, 2, pp. 429-444 (1978).

2. Farrell, M.J. "The measurement of productive effi- ciency", Journal of the Royal Statistical Society A, 120, pp. 253-281 (1957).

3. Banker, R.D., Charnes, A., and Cooper, W.W. "Some models for estimating technical and scale inefficiencies in data envelopment analysis", Management Science, 30(9), pp. 1031-1142 (1984).

4. Cooper, W.W., Park, K.S., and Pastor, J.T. "RAM: a range adjusted measure of on efficiency for use with additive models, and relations to other models and measures in DEA", Journal of Productivity Analysis, 11, pp. 5-24 (1999). 
5. Sueyoshi, T. "DEA non-parametric ranking test and index measurement: Slack-adjust DEA and an application to Japanese agriculture cooperative", Omega, 27, pp. 315-326 (1999).

6. Seiford, L.M. and Thrall, R.M. "Recent developments in DEA, the mathematical programming approach to frontier analysis", Journal of Econometrics, 46, pp. 7-38 (1990).

7. Tone, K. "A slacks-based measure of efficiency in data envelopment analysis", European Journal of Operational Research, 130(3), pp. 498-509 (2001).

8. Deprins, D., Simar, L., and Tulkens, H. "Measuring labor efficiency in post offices", In The Performance of Public Enterprises, M. Marchand, P. Pestieau, Tulkens, H., (Eds), Elsevier Science Publishers, Amesterdam, pp. 243-267 (1984).

9. Sahoo, B.K. and Tone, K. "Non-parametric measurement of economies of scale and scope in noncompetitive environment with price uncertainty", Omega, 41, pp. 97-111 (2013).

10. Färe, R., Grosskopf, S., and Lovell, C.A.K., Measurement of Efficiency of Production, Boston: KluwerNijhoff Publishing Co., Inc. (1985).

11. Jamshidi, M., Sanei, M., Mahmoodirad, A., et al. "Uncertain RUSSEL data envelopment analysis model: A case study in Iranian banks", Journal of Intelligent \& Fuzzy Systems, 37(2), pp. 2937-2951 (2019).

12. Sengupta, J.K. "Efficiency measurement in stochastic input-output system", International Journal of Systems Science, 13, pp. 273-287 (1982).

13. Banker, R.D. "Maximum likelihood, consistency and data envelopment analysis. A statistical foundation", Management Science, 39, pp. 1265-1273 (1993).

14. Cooper, W.W., Huang, Z., Lelas, V., et al. "Chance constrained programming formulations for stochastic characterizations of efficiency and dominance in DEA", Journal of Productivity Analysis, 9, pp. 53-79 (1998).

15. Grosskopf, S. "Statistical inference and nonparametric efficiency: a selective survey", Journal of Productivity Analysis, 7, pp. 161-176 (1996).

16. Olsen, O. and Petersen, N.C. "Chance constrained efficiency evaluation", Management Science, 131, pp. 442-457 (1995).

17. Cooper, W.W., Park, K.S., and Yu, G. "Idea and AR-IDEA: models for dealing with imprecise data in DEA", Management Science, 45, pp. 597-607 (1999).

18. Cooper, W.W., Park, K.S., and Yu, G. "An illustrative application of idea (imprecise data envelopment analysis) to Korean mobile telecommunication company", Operations Research, 49, pp. 807-820 (2001).

19. Cooper, W.W., Park, K.S., and Yu, G. "IDEA (imprecise data envelopment analysis) with CMDs (column maximum decision making units)", The Journal of the Operational Research Society, 52, pp. 176-181 (2001).

20. Kao, C. and Liu, S. "Fuzzy efficiency measures in data envelopment analysis", Fuzzy Sets and Systems, 113, pp. $427-437$ (2000).
21. Entani, T., Maeda, Y., and Tanaka, H. "Dual models of interval DEA and its extension to interval data", European Journal of Operational Research, 136, pp. 32-45 (2002).

22. Zadeh, L.A. "Fuzzy sets as a basis for a theory of possibility", Fuzzy Sets and Systems, 1, pp. 3-28 (1978).

23. Guo, P. and Tanaka, H. "Fuzzy DEA: a perceptual evaluation method", Fuzzy Sets and System, 119, pp. 149-160 (2001).

24. Lertworasirikul, S., Fang, S., Joines, J.A., et al. "Fuzzy data envelopment analysis (DEA): A possibility approach", Fuzzy Sets and Systems, 139, pp. 379-394 (2003).

25. Liu, B., Uncertain Theory, Springer, Berlin, Germany, 2nd Edn. (2007).

26. Jamshidi, M., Sanei, M., Mahmoodirad, A., et al. "Uncertain SBM data envelopment analysis model: A case study in Iranian banks", International Journal of Finance and Economics, 26(2), pp. 2674-2689 (2021). doi.org/10.1002/ijfe.1927

27. Mahmoodirad, A., Dehghan, R., and Niroomand, S. "Modelling linear fractional transportation problem in belief degree-based uncertain environment", Journal of Experimental \& Theoretical Intelligence, 31(3), pp. 393-408 (2018).

28. Liu, B., Uncertain Theory, Springer, Berlin, Germany, 4th Edn. (2015).

29. Wen, M., Guo, L., Kang, R., et al. "Data envelopment analysis with uncertain inputs and outputs", Journal of Applied Mathematics, 2014, pp. 1-7, Article ID 307108 (2014). http://dx.doi.org/10.1155/2014/ 307108

30. Wen, M., Qin, Z., Kang, R., et al., Sensitivity and Stability Analysis of the Additive Model in Uncertain Data Envelopment Analysis, Springer-Verlag Berlin Heidelberg (2014).

31. Wen, M., Zhang, Q., Kang, R., et al. "Some new ranking criteria in data envelopment analysis under uncertain environment", Computer \& Industrial Engineering, 110, pp. 498-504 (2017).

32. Lio, W. and Liu, B. "Uncertain data envelopment analysis with imprecisely observed inputs and outputs", Fuzzy Optimization and Decision Making, 17(3), pp. 357-373 (2017).

33. Mahmoodirad, A., Niroomand, S., and Hosseinzadeh Lotfi, F. "An effective solution approach for multiobjective fractional fixed charge problem with fuzzy parameters", Scientia Iranica, 27(4), pp. 2057-2068 (2020).

34. Majumder, S., Kundu, P., Kar, S., et al. "Uncertain multi-objective multi-item fixed charge solid transportation problem with budget constraint", Soft Computing, 23, pp. 3279-3301 (2020).

35. Lio, W. and Liu, B. "Residual and confidence interval for uncertain regression model with imprecise observations", Journal of Intelligent \& Fuzzy Systems, 35(2), pp. 2573-2583 (2018). 
36. Chen, W., Wang, Y., Gupta, P., et al. "A novel hybrid heuristic algorithm for a new uncertain meanvariance-skewness portfolio selection model with real constraints", Applied Intelligence, 48, pp. 2996-3018 (2018).

37. Zhai, J. and Bai, M. "Mean-variance model for portfolio optimization with background risk based on uncertainty theory", International Journal of General Systems, 47(3), pp. 294-312 (2018).

38. Dalman, H. "Entropy-based multi-item solid transportation problems with uncertain variables", Soft Computing, 23, pp. 5931-5943 (2018).

39. Zhang, B., Peng, J., Li, S., et al. "Fixed charge solid transportation problem in uncertain environment and its algorithm", Computer \& Industrial Engineering, 102, pp. 186-197 (2016).

40. Liu, B. "Some research problems in uncertainty theory", Journal of Uncertain System, 3(1), pp. 3-10 (2009).

41. Chen, X. and Dai, W. "Maximum entropy principle for uncertain variables", International Journal of Fuzzy Systems, 13(3), pp. 232-236 (2011).

42. Dai, W. and Chen, X. "Entropy of function of uncertain variables", Mathematical and Computer Modelling, 55(3), pp. 754-760 (2012).

43. Liu, B. "Why is there a need for uncertainty theory", Journal of Uncertain Systems, 6, pp. 3-10 (2012).

44. Peng, Z.X. and Iwamura, K. "Some properties of product uncertain measure", Journal of Uncertain Systems, 6(4), pp. 263-269 (2012).

45. Kolmogorov, A.N., Grundbegrie der Wahrscheinlichkeitsrechnung, Julius Springer, Berlin (1933).

46. Shannon, C.E. and Weaver, W., The Mathematical Theory of Communication, University of Illinois Press, Urbana (1949).
47. Liu, B., Uncertainty Theory: A Branch of Mathematics for Modeling Human Uncertainty, Springer Verlag, Berlin (2010).

48. Fubini, G. "Sugli integral multipli", Rom. Acc. L. Rend., 16(1), pp. 608-614 (1907).

\section{Biographies}

Mohammad Jamshidi is a PHD candidate of Applied Mathematics (Operations Research) at the Central Tehran Branch of Islamic Azad University, Iran. His research interests include data envelopment analysis and uncertainty theory. He has published research articles in international journals of mathematics and industrial engineering.

Masoud Sanei is an Associate Professor in the Department of Applied Mathematics, Islamic Azad University, Central Tehran Branch, Iran. He received his $\mathrm{PhD}$ degree in Applied Mathematics (Operations Research) from Islamic Azad University, Science and Research Branch, Tehran, in 2004. His research interests are in the areas of operations research such as data envelopment analysis, uncertainty theory, and supply chain management. He has several papers in journals and conference proceedings.

Ali Mahmoodirad is an Associate Professor of Applied Mathematics (Operations Research) at the Masjed-Sleiman Branch of Islamic Azad University in Iran. His research interests include fuzzy mathematical programming, uncertainty theory, supply chain management, and assembly line balancing. He has published research articles in international journals of mathematics and industrial engineering. 